TRANSACTIONS OF THE

AMERICAN MATHEMATICAL SOCIETY

Volume 353, Number 6, Pages 2193-2214

S 0002-9947(01)02701-5

Article electronically published on January 29, 2001

\title{
SIMPLE HOLONOMIC MODULES OVER RINGS OF DIFFERENTIAL OPERATORS WITH REGULAR COEFFICIENTS OF KRULL DIMENSION 2
}

\author{
V. BAVULA AND F. VAN OYSTAEYEN
}

\begin{abstract}
Let $K$ be an algebraically closed field of characteristic zero. Let $\Lambda$ be the ring of ( $K$-linear) differential operators with coefficients from a regular commutative affine domain of Krull dimension 2 which is the tensor product of two regular commutative affine domains of Krull dimension 1. Simple holonomic $\Lambda$-modules are described. Let a $K$-algebra $D$ be a regular affine commutative domain of Krull dimension 1 and $\mathcal{D}(D)$ be the ring of differential operators with coefficients from $D$. We classify (up to irreducible elements of a certain Euclidean domain) simple $\mathcal{D}(D)$-modules (the field $K$ is not necessarily algebraically closed).
\end{abstract}

\section{INTRODUCTION}

Let $K$ be an algebraically closed field of characteristic zero and let an algebra $R$ be a regular commutative affine domain of Krull dimension $\mathcal{K}(R)=2$. Let $\mathcal{D}(R)$ be the ring of ( $K$-linear) differential operators with coefficients from $R$. The ring $\mathcal{D}(R)$ is a simple Noetherian affine algebra that coincides with its subalgebra, the derivation ring, $\Delta(R)$, this being the ring generated by $R$ and its derivations. The Gelfand-Kirillov dimension of a simple $\mathcal{D}(R)$-module is either 2 or 3 . In the first case such a $\mathcal{D}(R)$-module is called holonomic (a definition of holonomic module over rings of differential operators is given in Section 2).

The aim of the present paper is to describe the simple holonomic $\mathcal{D}(R)$-modules in case the algebra $R=D_{1} \otimes D_{2}$ is the tensor product of regular commutative affine domains of Krull dimension 1. Observe that $\mathcal{D}\left(D_{1} \otimes D_{2}\right) \simeq \mathcal{D}\left(D_{1}\right) \otimes \mathcal{D}\left(D_{2}\right)$ (Lemma 2.5) and that the second Weyl algebra $A_{2}=A_{1} \otimes A_{1}$ is an example of the ring $\mathcal{D}\left(D_{1} \otimes D_{2}\right)$. The simple holonomic modules over the second Weyl algebra (and other popular simple generalized Weyl algebras of Gelfand-Kirillov dimension 4) were classified in BVO2. The present paper can be considered as a further development and extension of [BVO2], Bl1, Bl2, Bl3], [Bav1, Bav2, Bav3] and BVO1]. Stafford first gives examples of simple non-holonomic modules over the Weyl algebras [St. Later Bernstein and Lunts $\mathrm{BeLu}$, [Lu, and Coutinho $\mathrm{Co}$ construct more sophisticated examples of simple non-holonomic modules over the Weyl algebras.

Received by the editors September 15, 1998 and, in revised form, March 23, 2000.

2000 Mathematics Subject Classification. Primary 16S32, 32C38, 13N10.

The first author was supported by a grant of the University of Antwerp as a research fellow at U.I.A. 
Definitions and basic results on differential operators, Gelfand-Kirillov dimension and localizations are collected in Section 2 (for more details the reader is referred to [Bj], $\mathrm{Bor}],[\mathrm{KL}]$ and $[\mathrm{MR}]$ ).

In Section 3 we provide a description of simple modules over the $\operatorname{ring} \mathcal{D}(D)$ of differential operators with coefficients from a regular commutative affine domain $D$ of Krull dimension 1. In geometric language simple modules are classified over the ring of differential operators on a smooth irreducible algebraic curve. We use the approach of Block, Bl3], where the simple modules over the differential operator ring (the Ore extension) $T[X ; \delta]$ were classified under a "geometrical" condition ((3.1.1), [Bl3]) on the Dedekind domain $T$ and the derivation $\delta$ of $T$. Recently, these results were extended in [Bav3] to an arbitrary Ore extension $T[X ; \sigma, \delta]$, where $\sigma \in$ Aut $T$ and $\delta$ is a $\sigma$-derivation of a Dedekind domain $T$. It is interesting to observe that the "nontrivial" (see below) holonomic $\mathcal{D}\left(R=D_{1} \otimes D_{2}\right)$-modules arise exactly in the case where a noncommutative analog of the condition (3.1.1) of [B]3 fails. Note that every simple $\mathcal{D}(D)$-module is holonomic (i.e. has Gelfand-Kirillov dimension 1).

Let $M_{i}(i=1,2)$ be a simple $\mathcal{D}\left(D_{i}\right)$-module. Then the tensor product $M_{1} \otimes M_{2}$ is a simple holonomic $\mathcal{D}\left(D_{1}\right) \otimes \mathcal{D}\left(D_{2}\right)$-module and two such modules are isomorphic, $M_{1} \otimes M_{2} \simeq M_{1}^{\prime} \otimes M_{2}^{\prime}$, iff $M_{1} \simeq M_{1}^{\prime}$ and $M_{2} \simeq M_{2}^{\prime}$. Therefore, the set $\hat{\mathcal{D}}\left(D_{1} \otimes D_{2}\right)$ (holonomic) of isoclasses of simple holonomic modules contains the subset $\hat{\mathcal{D}}\left(D_{1}\right) \otimes \hat{\mathcal{D}}\left(D_{2}\right)$, the "trivial" holonimic modules. The "nontrivial" ones, i.e. $\hat{\mathcal{D}}\left(D_{1} \otimes D_{2}\right)$ (holonomic) $\backslash \hat{\mathcal{D}}\left(D_{1}\right) \otimes \hat{\mathcal{D}}\left(D_{2}\right)$, are described in Sections 4 and 5 .

In Section 4, the "nontrivial" holonomic modules are described in a special case of the ring $\Lambda:=\mathcal{D}\left(D_{1}\right) \otimes \mathcal{D}\left(D_{2}\right)$ where the second tensor term is isomorphic to a differential operator ring $\mathcal{D}\left(D_{2}\right)=D_{2}[X ; \delta]$. Let a skew field $k$ be the full quotient ring of $\mathcal{D}\left(D_{1}\right)$. Let $\mathcal{A}$ be the (two-sided) localization of $\Lambda$ at $\mathcal{D}\left(D_{1}\right)_{*}:=\mathcal{D}\left(D_{1}\right) \backslash\{0\}$ i.e.

$$
\mathcal{A}=k \otimes \mathcal{D}\left(D_{2}\right)=k \otimes D_{2}[X ; \delta] .
$$

The first main result (Theorem 4.9) states that the map

$$
\hat{\Lambda} \text { (holonomic) } \backslash \hat{\mathcal{D}}\left(D_{1}\right) \otimes \hat{\mathcal{D}}\left(D_{2}\right) \rightarrow \hat{\mathcal{A}}(k \text {-fin.dim }),[M] \rightarrow\left[\mathcal{A} \otimes_{\Lambda} M\right],
$$

is bijective with inverse $[N] \rightarrow\left[\operatorname{Soc}_{\Lambda} N\right]$, where $\hat{\mathcal{A}}(k$-fin.dim) is the set of isoclasses of simple $\mathcal{A}$-modules which are a finite dimensional left vector space over the skew field $k$.

The second main result of Section 4 (Corollary 4.11) provides a holonomicity criterion.

Let $M$ be a (nonzero) simple $\Lambda$-module and let $\tilde{M}=\mathcal{A} \otimes_{\Lambda} M$. Then

1. $\tilde{M}=0 \Leftrightarrow[M] \in \hat{\mathcal{D}}\left(D_{1}\right) \otimes \hat{\mathcal{D}}\left(D_{2}\right)$;

2. $1 \leq \operatorname{dim}_{k} \hat{M}<\infty \Leftrightarrow[M] \in \hat{\Lambda}$ (holonomic) $\backslash \hat{\mathcal{D}}\left(D_{1}\right) \otimes \hat{\mathcal{D}}\left(D_{2}\right)$;

3. $\operatorname{dim}_{k} \tilde{M}=\infty \Leftrightarrow[M] \in \hat{\Lambda}$ (non-holonomic).

Hence, $M$ is holonomic (resp. non-holonomic) iff $\operatorname{dim}_{k} \tilde{M}<\infty$ (resp. $M$ contains a free $\mathcal{D}\left(D_{1}\right) \otimes K[X]$-module of rank 1$)$.

Corollary 4.12 yields a presentation of every element $[M] \in \hat{\Lambda}$ (holonomic) $\backslash \hat{\mathcal{D}}\left(D_{1}\right)$ $\otimes \hat{\mathcal{D}}\left(D_{2}\right)$ as a factor module of the $\Lambda$-module $\Lambda$.

The idea behind the description of $\hat{\mathcal{D}}\left(D_{1} \otimes D_{2}\right)$ (holonomic) $\backslash \hat{\mathcal{D}}\left(D_{1}\right) \otimes \hat{\mathcal{D}}\left(D_{2}\right)$ is to reduce to the case of Section 4 .

In Section 5 the global results are obtained. 
In Section 6, for the ring of differential operators $\mathcal{D}(R)$ with coefficients from a regular commutative affine domain $R$ of Krull dimension 2 a holonomicity criterion (Theorem 6.1) is established.

In the paper, module means a left module.

\section{Preliminary Results}

Let $K$ be a field of characteristic zero and $R$ be a commutative $K$-algebra. A ring of (K-linear) differential operators $\mathcal{D}(R)$ on $R$ is defined as $\mathcal{D}(R)=\bigcup_{i=0}^{\infty} \mathcal{D}^{i}(R)$ where $\mathcal{D}^{0}(R)=\left\{u \in \operatorname{End}_{K}(R): u r-r u=0\right.$, for all $\left.r \in R\right\}=\operatorname{End}_{R}(R) \simeq R$,

$$
\mathcal{D}^{i}(R)=\left\{u \in \operatorname{End}_{K}(R): u r-r u \in \mathcal{D}^{i-1}(R), \text { for all } r \in R\right\} .
$$

Note that the $\left\{\mathcal{D}^{i}(R)\right\}$ defines a filtration for $\mathcal{D}(R)$. We say that an element $u \in \mathcal{D}^{i}(R) \backslash \mathcal{D}^{i-1}(R)$ has order $i$. The subalgebra $\Delta(R)$ of $\operatorname{End}_{K}(R)$ generated by $R \equiv \operatorname{End}_{R}(R)$ and by the set $\operatorname{Der}_{K}(R)$ of all $K$-derivations of $R$ is called the derivation ring of $R$. The derivation ring $\Delta(R)$ is the subring of $\mathcal{D}(R)$.

Let the algebra $R$ be a regular commutative affine domain of Krull dimension $n<\infty$. In geometric terms, $R$ is the coordinate ring $\mathcal{O}(X)$ of a smooth irreducible affine variety $X$ of dimension $n$. Then

- $\operatorname{Der}_{K}(R)$ is a finitely generated projective $R$-module of rank $n$;

- $\mathcal{D}(R)=\Delta(R)$;

- $\mathcal{D}(R)$ is a simple (left and right) Noetherian domain;

- gld $\mathcal{D}(R)=\mathcal{K}(\mathcal{D}(R))=\mathrm{GK}(\mathcal{D}(R)) / 2=\mathcal{K}(R)=n$ (where gld, $\mathcal{K}$ and $\mathrm{GK}$ stand for the global, Krull and Gelfand-Kirillov dimension respectively);

- $\mathrm{GK}(M) \geq n$ for any nonzero finitely generated $\mathcal{D}(R)$-module $M$;

- if $S$ is a multiplicatively closed subset of $R$, then $S$ is a (left and right) Ore set of $\mathcal{D}(R)$ and $\mathcal{D}\left(S^{-1} R\right)=S^{-1} \mathcal{D}(R)$;

- $\mathcal{D}(R)=\Delta(R)$ is an almost centralizing extension of $R$;

- $\Delta(R)$ is a somewhat commutative algebra;

- $\Delta(R)$ satisfies the Nullstelenzatz over $K$. So, $\operatorname{End}_{\Delta(R)}(M)=K$ for every simple $\Delta(R)$-module $M$ since $K$ is an algebraically closed field;

- the associative graded ring $\operatorname{gr} \mathcal{D}(R)=\bigoplus \mathcal{D}^{i}(R) / \mathcal{D}^{i-1}(R)$ is a commutative domain.

For the proofs the reader is referred to MR, Chapter 15.

Definition. A finitely generated $\mathcal{D}(R)$-module $M$ is called holonomic if it has Gelfand-Kirillov dimension

$$
\mathrm{GK}(M)=\mathrm{GK}(\mathcal{D}(R)) / 2=n .
$$

Example. Let $P_{n}=K\left[X_{1}, \ldots, X_{n}\right]$ be a polynomial ring in $n$ indeterminates.

$$
\mathcal{D}\left(P_{n}\right)=K\left[X_{1}, \ldots, X_{n}, \partial / \partial X_{1}, \ldots, \partial / \partial X_{n}\right]
$$

is the $n$th Weyl algebra, $A_{n}=A_{n}(K)$. Clearly, $A_{n}=A_{1} \otimes \cdots \otimes A_{1}$ ( $n$ times).

The following technical lemma will be used frequently in the paper (15.2.13 and 15.3.2, [MR $]$.

Lemma 2.1. Let $R$ be a regular commutative $K$-domain of Krull dimension $n$.

1. Let $\mathbf{m}$ be a maximal ideal of $R$. Then there exists $c=c(\mathbf{m}) \in R \backslash \mathbf{m}$ such that

$$
\mathcal{D}(R)_{c}=R_{c}\left[X_{1} ; \partial / \partial Y_{1}\right] \cdots\left[X_{n} ; \partial / \partial Y_{n}\right]
$$


an iterated Ore extension for which $X_{i} X_{j}=X_{j} X_{i}$ for $1 \leq i, j \leq n$, and $\left\{Y_{1}, \ldots, Y_{n}\right\}$ is a transcendence basis for the quotient field of $R$; the algebra $\mathcal{D}(R)_{c}$ contains the $n$th Weyl algebra

$$
\begin{aligned}
A_{n} & =K\left[Y_{1}, \ldots, Y_{n}\right]\left[X_{1} ; \partial / \partial Y_{1}\right] \cdots\left[X_{n} ; \partial / \partial Y_{n}\right] \\
& =K\left[Y_{1}, \ldots, Y_{n}, \partial / \partial Y_{1}, \ldots, \partial / \partial Y_{n}\right]
\end{aligned}
$$

and is a (left and right) finitely generated $A_{n}$-module;

2. there is a finite subset $\left\{c_{1}, \ldots, c_{s}\right\}$ of $\{c(\mathbf{m}) \mid \mathbf{m}$ is a maximal ideal of $R\}$ (of elements as above) such that the natural ring monomorphism $\mathcal{D}(R) \rightarrow$ $\prod_{i=1}^{s} \mathcal{D}(R)_{c_{i}}$ is (left and right) faithfully flat.

The $K$-algebra $R$ is affine, so let $\left\{R_{i}, i \geq 0\right\}\left(R_{0}=K\right)$ be a finite dimensional filtration of $R$ such that the associated graded algebra gr $R=\bigoplus R_{i} / R_{i-1}$ is affine. In particular, every standard filtration of $R$ satisfies this property. The $\mathcal{D}(R)=\Delta(R)$ is an almost centralizing extension of $R\left(15.1 .20\right.$, $\mathrm{MR}$, and $\operatorname{Der}_{K}(R)$ is a finitely generated $R$-module). By (8.6.7, [MR]), the algebra $\Delta(R)$ has a finite dimensional filtration $\mathcal{F}=\left\{\Delta_{i}(R), i \geq 0\right\}\left(\Delta_{0}(R)=K\right)$ such that $\operatorname{gr}_{\mathcal{F}} \Delta(R)$ is a commutative affine algebra (hence Noetherian), i.e., by definition, $\Delta(R)$ is a somewhat commutative algebra. The graded $K$-algebra $\operatorname{gr}_{\mathcal{F}} \Delta(R)$ is generated by homogeneous elements, say $x_{1}, \ldots, x_{t}$, of positive graded degrees $k_{1}, \ldots, k_{t}$, respectively. Let $k$ be the least common multiple of $\left\{k_{i}\right\}$.

A filtration $\Gamma=\left\{\Gamma_{i}, i \geq 0\right\}$ of a $\Delta(R)$-module $M=\bigcup_{i=0}^{\infty} \Gamma_{i}$ is called good if the associated graded $\operatorname{gr}_{\mathcal{F}} \Delta(R)$-module $\operatorname{gr}_{\Gamma} M$ is finitely generated. $\mathrm{A} \Delta(R)$ module $M$ has a good filtration iff it is finitely generated; and if $\left\{\Gamma_{i}\right\}$ and $\left\{\Omega_{i}\right\}$ are two good filtrations on $M$, then there exists a natural number $j$ such that $\Gamma_{i} \subseteq \Omega_{i+j}$ and $\Omega_{i} \subseteq \Gamma_{i+j}$ for all $i$. If a $\Delta(R)$-module $M$ is finitely generated and $M_{0}$ is a finite dimensional generating subspace of $M$, then the standard filtration $\left\{\Gamma_{i}=\Delta_{i}(R) M_{0}\right\}$ is good (see $[\mathrm{Bj}]$ or [LVO for details). The following lemma is well known for specialists (see, for example, [Bav4]).

Lemma 2.2. Let $M$ be a finitely generated $\Delta(R)$-module with good filtration $\Gamma=$ $\left\{\Gamma_{i}\right\}$. Then

1. there exist $k$ polynomials $\gamma_{0}, \ldots, \gamma_{k-1} \in \mathbf{Q}[t]$ with coefficients from $\left[k^{\mathrm{GK}(M)} \mathrm{GK}(M) !\right]^{-1} \mathbf{Z}$ such that

$$
\operatorname{dim} \Gamma_{i}=\gamma_{j}(i) \text { for all } i>>0 \text { and } j \equiv i(\bmod k) ;
$$

2. the polynomials $\gamma_{j}$ have the same degree $\operatorname{GK}(M)$ and the same leading coefficients $e(M) / \operatorname{GK}(M)$ ! where $e(M)$ is called the multiplicity of $M$. The multiplicity $e(M)$ does not depend on the choice of good filtration $\Gamma$.

Let $0 \rightarrow N \rightarrow M \rightarrow L \rightarrow 0$ be an exact sequence of finitely generated $\Delta(R)$ modules and let $\Gamma=\left\{\Gamma_{i}\right\}$ be a good filtration on $M$. Then $\Gamma^{\prime}=\left\{\Gamma_{i}{ }^{\prime}=\Gamma_{i} \cap N\right\}$ and $\Gamma^{\prime \prime}=\left\{\Gamma_{i}^{\prime \prime}=\left(\Gamma_{i}+N\right) / N\right\}$ are filtrations on $N$ and $L$ respectively such that the sequence of $\operatorname{gr}_{\mathcal{F}} \Delta(R)$-modules

$$
0 \rightarrow \operatorname{gr}_{\Gamma^{\prime}}(N) \rightarrow \operatorname{gr}_{\Gamma}(M) \rightarrow \operatorname{gr}_{\Gamma^{\prime \prime}}(L) \rightarrow 0
$$

is exact. The ring $\operatorname{gr}_{\mathcal{F}} \Delta(R)$ is Noetherian and the $\operatorname{gr}_{\mathcal{F}} \Delta(R)$-module $\operatorname{gr}_{\Gamma}(M)$ is finitely generated, so the $\operatorname{gr}_{\mathcal{F}} \Delta(R)$-modules $\operatorname{gr}_{\Gamma^{\prime}}(N)$ and $\operatorname{gr}_{\Gamma^{\prime \prime}}(L)$ are finitely generated, i.e. the filtrations $\Gamma^{\prime}$ and $\Gamma^{\prime \prime}$ are good and we have

$$
\operatorname{dim} \Gamma_{i}=\operatorname{dim} \Gamma_{i}{ }^{\prime}+\operatorname{dim} \Gamma_{i}^{\prime \prime},
$$


hence, by Lemma 2.2 ,

$$
\mathrm{GK}(M)=\max \{\mathrm{GK}(N), \mathrm{GK}(L)\},
$$

and if $\mathrm{GK}(M)=\mathrm{GK}(N)=\mathrm{GK}(L)$, then

$$
e(M)=e(N)+e(L)
$$

A finitely generated $\Delta(R)$-module $M$ is called holonomic if GK $M=\mathrm{GK}(\Delta(R)) / 2$ $=\mathcal{K}(R)$.

- Every holonomic $\Delta(R)$-module has finite length.

(For example, it follows from (2.3) and Lemma 2.2.) Let $0 \neq c \in R$ and $M$ be an $R$-module. We denote by $M_{c}$ the localization of $M$ at the powers of the element c, i.e. $M_{c}=S^{-1} M$ where $S=\left\{c^{i}, i \geq 0\right\}$. The module $M$ is called $c$-torsionfree (resp. $c$-torsion) if the map $c_{M}: M \rightarrow M, m \rightarrow c m$, is injective (resp. $S^{-1} M=0$ ).

Denote by $\mathbf{N}=\{0,1, \ldots\}$ and $\mathbf{R}$ the set of natural and real numbers. For a function $f: \mathbf{N} \rightarrow \mathbf{N}$ its degree is defined as follows:

$$
\gamma(f):=\inf \left\{d \in \mathbf{R}: f(n) \leq n^{d} \text { for sufficiently large } n>>0\right\} .
$$

Proposition 2.3. Let $c$ be a nonzero element of $R$ and let $M$ be a finitely generated c-torsionfree $\Delta(R)$-module. Then $\mathrm{GK}_{\Delta(R)} M=\mathrm{GK}_{\Delta(R)_{c}} M_{c}$.

Proof. The $\Delta(R)$-module $M$ is $c$-torsionfree, thus the map $M \rightarrow M_{c}, m \rightarrow m / 1$, is a monomorphism of $\Delta(R)$-modules. So, $\mathrm{GK}_{\Delta(R)} M \leq \mathrm{GK}_{\Delta(R)_{c}} M_{c}$.

Conversely, fix a finite dimensional filtration $\mathcal{F}=\left\{\Delta_{i}(R), i \geq 0\right\}$ of the algebra $\Delta(R)$ as above, i.e. the associated graded algebra $\operatorname{gr}_{\mathcal{F}} \Delta(R)$ is commutative affine. Let $M_{0}$ be a finite dimensional generating subspace of the $\Delta(R)$-module $M$. The $M$ is equipped with the good filtration $\left\{M_{i}=\Delta_{i}(R) M_{0}\right\}$ and $\mathrm{GK}_{\Delta(R)} M=$ $\gamma\left(\operatorname{dim} M_{i}\right)$. Let $A_{1} \ni 1$ be a finite dimensional subspace of algebra generators of $\Delta(R)$. Then $\Delta(R)_{c}$ has the standard filtration $\left\{B_{i}=B_{1}^{i}, i \geq 0\right\}$ where $B_{1}=$ $A_{1}+K c^{-1}$. The $\Delta(R)_{c}$-module $M_{c}$ has the standard filtration $\left\{\left(M_{c}\right)_{i}=B_{i} M_{0}\right\}$ and $\mathrm{GK}_{\Delta(R)_{c}} M_{c}=\gamma\left(\operatorname{dim}\left(M_{c}\right)_{i}\right)$. Using 15.1.17, MR, we can find natural numbers $\alpha$ and $\beta$ such that $c^{\alpha i} B_{i} \subseteq \Delta_{\beta i}$ for all $i$. Now, $c^{\alpha i}\left(M_{c}\right)_{i}=c^{\alpha i} B_{i} M_{0} \subseteq \Delta_{\beta i} M_{0}=M_{\beta i}$ and $\operatorname{dim}\left(M_{c}\right)_{i} \leq \operatorname{dim} M_{\beta i}$, hence $\mathrm{GK}_{\Delta(R)_{c}} M_{c} \leq \mathrm{GK}_{\Delta(R)} M$.

Theorem 2.4. Let $M$ be a finitely generated $\Delta(R)$-module and let $\Delta(R) \rightarrow$ $\prod_{i=1}^{s} \Delta(R)_{c_{i}}$ be a faithfully flat extension from Lemma 2.1. Then

1. $\mathrm{GK}_{\Delta(R)} M=\max \left\{\mathrm{GK}_{\Delta(R)_{c_{i}}} M_{c_{i}}\right\}$;

2. $M$ is a holonomic $\Delta(R)$-module iff each nonzero $M_{c_{i}}$ is a holonomic $\Delta(R)_{c_{i}}$ module;

3. $N$ is a holonomic $\Delta(R)_{c_{i}}$-module iff $N$ is a holonomic module over the $n$th Weyl subalgebra $A_{n}^{(i)}$ from Lemma 2.1(1).

Proof. 1. Denote by $m$ the maximum in the statement of theorem. For a $\Delta(R)$ submodule $N$ of $M$ denote by $\bar{N}_{i}$ the image of $N$ under the map $M \rightarrow M_{c_{i}}$. Then, by Proposition 2.3, GK ${ }_{\Delta(R)} M \geq \mathrm{GK}_{\Delta(R)} \bar{M}_{i}=\mathrm{GK}_{\Delta(R)_{c_{i}}}\left(\bar{M}_{i}\right)_{c_{i}}=\mathrm{GK}_{\Delta(R)_{c_{i}}} M_{c_{i}}$, hence $\mathrm{GK}_{\Delta(R)} M \geq m$.

To prove the opposite inequality it suffices to prove the existence of a $\Delta(R)$ submodule $N$ of $M$ such that $\mathrm{GK}_{\Delta(R)} \bar{N}_{i}=\mathrm{GK}_{\Delta(R)} M$ for some $i$. Since then, by Proposition 2.3, GK ${ }_{\Delta(R)} \bar{N}_{i}=\mathrm{GK}_{\Delta(R)_{c_{i}}}\left(\bar{N}_{i}\right)_{c_{i}} \leq \mathrm{GK}_{\Delta(R)_{c_{i}}} M_{c_{i}}$, i.e. GK $\mathrm{GK}_{\Delta(R)} M$ $\leq m$. 
Let $n=n(M)$ be the number of nonzero maps $M \rightarrow M_{c_{i}}$. If $n=1$, i.e. $M \simeq \bar{M}_{i}$ for a unique $i$, then we can take $N=M$. Suppose $n>1$. Up to numeration we may suppose that first $n$ maps $\left\{M \rightarrow M_{c_{i}}, i=1, \ldots, n\right\}$ are nonzero. We have the exact sequences of $\Delta(R)$-modules: $0 \rightarrow K_{i} \rightarrow M \rightarrow \bar{M}_{i} \rightarrow 0, i=1, \ldots, n$, where $K_{i}$ is the kernel of the map $M \rightarrow M_{c_{i}}$. If there exists $i$ such that GK $\bar{M}_{i}=$ GK $M$, then we take $N=M$. Otherwise, GK $K_{i}=$ GK $M$ for all $i$. For the module $K_{1}$ the number $n\left(K_{1}\right)$ is less than $n$ (since $\bar{K}_{1}=0$ ), so the induction completes the argument.

$1 \Rightarrow 2$. Evident.

3. The $A_{n}^{(i)}$ is the affine subalgebra of the affine algebra $\Delta(R)_{c_{i}}$ such that $\Delta(R)_{c_{i}}$ is a finitely generated $A_{n}^{(i)}$-module (Lemma 2.1.(1)). Now, by Lemma 4.11, [BVO2], $\mathrm{GK}_{\Delta(R)_{c_{i}}} N=\mathrm{GK}_{A_{n}^{(i)}} N$ and the result follows.

Lemma 2.5. Let $R$ be a regular commutative affine domain of finite Krull dimension which is the tensor product $\bigotimes_{i=1}^{n} R_{i}$ of regular commutative affine domains $R_{i}$. Then $\mathcal{D}(R)=\bigotimes_{i=1}^{n} \mathcal{D}\left(R_{i}\right)$.

Proof. Observe $\mathcal{D}(R)=\Delta(R)$. It is sufficient to prove the statement in case $n=2$. Set $\Delta=\Delta(R), \Delta_{i}=\Delta\left(R_{i}\right), i=1,2$. Then $\Delta \supseteq \Delta_{1} \otimes \Delta_{2}$. Choose faithfully flat extensions $\Delta_{1} \rightarrow \prod_{i} \Delta_{1, c_{i}}, \Delta_{2} \rightarrow \prod_{j} \Delta_{2, t_{j}}$ as in Lemma 2.1. Then $\Delta_{1} \otimes \Delta_{2} \rightarrow$ $\prod_{i, j}\left(\Delta_{1}\right)_{c_{i}} \otimes\left(\Delta_{2}\right)_{t_{j}}=\prod_{i, j}\left(\Delta_{1} \otimes \Delta_{2}\right)_{c_{i} t_{j}}$ is the faithfully flat extension (if $M$ is a nonzero $\Delta_{1} \otimes \Delta_{2}$-module, then there exists $i$ such that $M_{c_{i}} \neq 0$ and then there exists $j=j(i)$ such that $\left.M_{c_{i} t_{j}} \neq 0\right)$. Evidently,

$$
\Delta_{c_{i} t_{j}}=\Delta\left(R_{c_{i} t_{j}}\right)=\Delta\left(\left(R_{1}\right)_{c_{i}}\right) \otimes \Delta\left(\left(R_{2}\right)_{t_{j}}\right)=\left(\Delta_{1} \otimes \Delta_{2}\right)_{c_{i} t_{j}}
$$

for $i, j$. We have $\Delta \supseteq \Delta_{1} \otimes \Delta_{2}$ with $\prod \Delta_{c_{i} t_{j}}=\prod\left(\Delta_{1} \otimes \Delta_{2}\right)_{c_{i} t_{j}}$, since $\Delta_{1} \otimes \Delta_{2} \rightarrow$ $\prod_{i, j}\left(\Delta_{1} \otimes \Delta_{2}\right)_{c_{i} t_{j}}$ is a faithfully flat extension, we conclude that $\Delta=\Delta_{1} \otimes \Delta_{2}$.

Let $A$ be a ring and let $B=S^{-1} A$ be the left (Ore) localization of the ring $A$ at an Ore set $S \ni 1$ of $A$. We have the natural ring homomorphism $A \rightarrow B, a \rightarrow a / 1$, which, in general, is not a monomorphism. For a left ideal $\mathbf{m}$ of $B$ we denote by $A \cap \mathbf{m}$ the inverse image of $\mathbf{m}$ in $A$. The localization defines the localization functor

$$
S^{-1}: A \text {-mod } \rightarrow B \text {-mod, } \quad M \rightarrow S^{-1} M \simeq B \otimes_{A} M,
$$

from the category of $A$-modules to the category of $B$-modules. An $A$-module $M$ contains the $S$-torsion submodule

$$
\operatorname{tor}_{S}(M)=\{m \in M: s m=0 \text { for some } s=s(m) \in S\} .
$$

If the $A$-module $M$ is simple, then its localization $S^{-1} M$ is either zero $(\Leftrightarrow M=$ $\left.\operatorname{tor}_{S}(M)\right)$ or not $\left(\Leftrightarrow \operatorname{tor}_{S}(M)=0\right)$, in the last case $S^{-1} M$ is a simple $B$-module. Correspondingly, we say that a simple $A$-module is either $S$-torsion or $S$-torsionfree, i.e.

$$
\hat{A}=\hat{A}(S \text {-torsion }) \cup \hat{A}(S \text {-torsionfree }) .
$$

The sum of all simple submodules of an $A$-module $M$ is called the socle $\operatorname{Soc}_{A} M$ of $M$. It is the largest semisimple submodule of $M$. A $B$-module $N$ is called $A$ socle (or, socle, for short) provided $\operatorname{Soc}_{A} N \neq 0$. Denote by $\hat{B}$ ( $A$-socle) the set of isoclasses of simple $A$-socle $B$-modules. A submodule $M^{\prime}$ of $M$ is called essential if it intersects nontrivially each nonzero submodule of $M$. The following two lemmas are evident (see BVO2 for detail). 
Lemma 2.6. 1. The canonical map

$$
S^{-1}: \hat{A}(S \text {-torsionfree }) \rightarrow \hat{B}(A \text {-socle }),[M] \rightarrow\left[S^{-1} M\right],
$$

is a bijection with inverse $\operatorname{Soc}:[N] \rightarrow\left[\operatorname{Soc}_{A}(N)\right]$.

2. Each simple $S$-torsionfree A-module has the form

$$
M_{\mathbf{m}}:=A / A \cap \mathbf{m}
$$

for some left maximal ideal $\mathbf{m}$ of the ring $B$. Two such modules are isomorphic, $M_{\mathbf{m}} \simeq M_{\mathbf{n}}$, iff the $B$-modules $B / \mathbf{m}$ and $B / \mathbf{n}$ are isomorphic.

Write $L M A X(B)$ for the set of all left maximal ideals of $B$. A maximal left ideal $\mathbf{m}$ of the ring $B$ is called socle, resp. convenient, provided $\operatorname{Soc}_{A} M_{\mathbf{m}} \neq 0$ resp. $M_{\mathbf{m}}$ is a simple $A$-module and the sets of all such ideals are denoted by $L M A X . \operatorname{soc}(B)$ and $L M A X \cdot \operatorname{con}(B)$. Clearly, $L M A X \cdot \operatorname{con}(B) \subseteq L M A X \cdot \operatorname{soc}(B)$. In general, not every left maximal (resp. socle) ideal is socle (resp. convenient).

For a socle maximal left ideal $\mathbf{m}$ of $B$ let $J(\mathbf{m})$ be the smallest of the left ideals of $A$ strictly containing $A \cap \mathbf{m}$, then

$$
J(\mathbf{m}) / A \cap \mathbf{m}=\operatorname{Soc}_{A} M_{\mathbf{m}} .
$$

Since $S^{-1}(J(\mathbf{m}) / A \cap \mathbf{m})=S^{-1} \operatorname{Soc}{ }_{A} M_{\mathbf{m}}=B / \mathbf{m}$, the set

$$
\mathbf{a}(\mathbf{m}):=J(\mathbf{m}) \cap S
$$

is not empty.

Lemma 2.7. Let $\mathbf{m} \in L M A X . \operatorname{soc}(B)$ and $\alpha \in S$. The following are equivalent:

1. $\alpha \in \mathbf{a}(\mathbf{m})$;

2. $J(\mathbf{m})=A \alpha+A \cap \mathbf{m}$;

3. $M_{\mathbf{m} \alpha^{-1}}$ is a simple A-module;

4. $\mathbf{m} \alpha^{-1} \in L M A X \cdot \operatorname{con}(B)$.

\section{Classification of simple modules over Rings of Differential OPERATORS With REgUlaR COEFFICIENTS OF KRULL DIMENSION 1}

Let a $K$-algebra $D$ be a regular affine commutative domain of Krull dimension 1 over a field $K$ of characteristic zero (not necessarily algebraically closed). The ring $D$ is a Dedekind domain. The algebra $D$ can be seen as the coordinate ring of a smooth irreducible algebraic curve. Let $\Delta=\Delta(D)=\mathcal{D}(D)$ be the ring of differential operators with coefficients from $D$. In geometric terms, $\Delta$ is the ring of differential operators on a smooth irreducible algebraic curve. Observe that the algebra $\Delta$ is a simple affine Noetherian domain with Gelfand-Kirillov dimension 2 and Krull dimension 1. Using results of [Bl3], in this section we classify (up to the irreducible elements of a noncommutative Euclidean domain $B$, see below) the simple $\Delta$-modules (Theorems 3.4 and 3.6).

Let $\delta$ be a $K$-derivation of $D$ such that the Ore extension (or the differential operator ring) $A=D[X ; \delta]$ is a SIMPLE algebra. The first Weyl algebra $A_{1} \simeq$ $K[t][X ; d / d t]$ gives an example of the ring $A$. Denote by $l$ the field of fractions of $D$, i.e. $l=D_{*}^{-1} D$ where $D_{*}=D \backslash\{0\}$. Then the (two-sided) localization $B=D_{*}^{-1} A$ of $A$ at $D_{*}$ is the Ore extension $B=l[X ; \delta]$ with coefficients from the field $l$. By the ring monomorphism $A \rightarrow B, a \rightarrow a / 1$, we identify $A$ with its image in $B$ (the "new" $\delta$ is the unique extension of the $\delta$ from $D$ to $l$ ). The ring $B$ is a left and right Euclidean ring, hence, a left and right principal ideal domain. So, a $B$-module $N$ 
is simple iff $N \simeq B / B b$ for some irreducible element $b$ of $B$; and $B / B b \simeq B / B c$ iff $b$ and $c$ are similar (see Jac for details).

If $M$ is a simple $A$-module, then the localization $D_{*}^{-1} M=B \otimes_{A} M$ of $M$ at $D_{*}$ is either 0 or a (nonzero) simple $B$-module. We say that $M$ is $D$-torsion or $D$-torsionfree correspondingly. The set $\hat{A}$ is partitioned as

$$
\hat{A}=\hat{A}(D \text {-torsion }) \cup \hat{A}(D \text {-torsionfree }) \text {. }
$$

The field $K$ has characteristic zero, so the condition (3.1.1) of [Bl3] holds and there is no proper $\delta$-invariant ideal of the ring $D$ (since $A$ is simple). Let us recall the description of $\hat{A}$ following [Bl3]. Denote by Specm $D$ the set of prime ideals of $D$. Here "prime" means a nonzero prime (i.e. a maximal ideal).

Lemma 3.1 (4.1, Bl3]). The map

$$
\text { Specm } D \rightarrow \hat{A}(D \text {-torsion }), \mathbf{p} \rightarrow\left[A / A \mathbf{p} \simeq A \otimes_{D} D / \mathbf{p}\right],
$$

is a bijection.

Suppose $\mathbf{p}$ is a prime ideal of $D$. Let $\nu_{\mathbf{p}}$ denote the valuation of $l$ corresponding to $\mathbf{p}$, that is, if $d \in \mathbf{p}^{i} \backslash \mathbf{p}^{i-1}$, then $\nu_{\mathbf{p}} d=i$. The valuation ring $\left\{\alpha \in l \mid \nu_{\mathbf{p}} \alpha \geq 0\right\}$ coincides with the localization $D_{\mathbf{p}}$ of $D$ at $\mathbf{p}$ which is a local Dedekind domain with the maximal ideal $D_{\mathbf{p}} \mathbf{p}$. We identify the residue class field $D_{\mathbf{p}} / D_{\mathbf{p}} \mathbf{p}$ with $l_{\mathbf{p}}=D / \mathbf{p}\left(\equiv(D / \mathbf{p})_{\mathbf{p}}=D_{\mathbf{p}} / D_{\mathbf{p}} \mathbf{p}\right)$. We denote by $\eta_{\mathbf{p}}$ the canonical epimorphism $D_{\mathbf{p}} \rightarrow D_{\mathbf{p}} / D_{\mathbf{p}} \mathbf{p}=l_{\mathbf{p}}$.

The valuation $\nu_{\mathbf{p}}$ can be extended to a valuation on $B$, also denoted by $\nu_{\mathbf{p}}$ or by $\nu$ (for short), as follows: if $b=\sum b_{i} X^{i} \in B$, then (Lemma 3.1, Bl3])

$$
\nu_{\mathbf{p}} b=\min \left\{\nu_{\mathbf{p}} b_{i}-i \mid i \geq 0\right\} .
$$

Suppose $\mathbf{p}$ is a prime ideal of $D$. Pick $g \in \mathbf{p} \backslash \mathbf{p}^{2}$. For $b=\sum b_{i} X^{i} \in B$, R. Block ([Bl3], (3.2.1)) defines a polynomial $Q_{b}=Q_{b}(t)=Q_{\mathbf{p}, g, b}(t) \in l_{\mathbf{p}}[t]$ by

$$
Q_{\mathbf{p}, g, b}(t)=\sum_{i \geq 0} \eta_{\mathbf{p}}\left(g^{-\nu_{\mathbf{p}} b-i} b_{i}(\delta g)^{i}\right) t(t-1) \cdots(t-i+1)
$$

The polynomial above is called the indicial polynomial of $b$ relative to $\mathbf{p}, g$ (or at $\mathbf{p}, g$ ). The normalized indicial polynomial $\bar{Q}_{\mathbf{p}, b}$ of $b$ relative to $\mathbf{p}$, which is obtained by dividing $Q_{\mathbf{p}, g, b}$ by its leading coefficient, is independent of the choice of $g$ (Lemma 3.2, [B13]). The roots of $Q_{\mathbf{p}, g, b}$ are called the indicial roots of $b$ relative to $\mathbf{p}$ (this being independent of $g$ ). The element $b$ is preserving relative to $\mathbf{p}$ if there is no negative integer indicial root relative to $\mathbf{p}$. We shall also say that $b$ is preserving if it is preserving relative to $\mathbf{p}$, for every prime $\mathbf{p}$ of $D$.

If $b=\sum b_{i} X^{i} \in B$ of degree $k>0$, a prime $\mathbf{p}$ is called the special prime of $b$ if $\nu_{\mathbf{p}} b=\nu_{\mathbf{p}} b_{i}-i$ for some $i<k$. The element $b$ has only finitely many special primes. If $\mathbf{p}$ is not a special prime of $b$, then $b$ is preserving relative to $\mathbf{p}$. Hence the property of $b$ being preserving depends on only finitely many primes (see 3.4 , [Bl3] for details).

Theorem $3.2\left(4.4,[\mathrm{Bl} 3)\right.$. Let $b=\sum b_{i} X^{i} \in B$ be an irreducible preserving element. Then $A / A \cap B b$ is a simple D-torsionfree A-module. Up to isomorphism every simple $D$-torsionfree $A$-module arises in this way and two such $A$-modules are isomorphic, $A / A \cap B b \simeq A / A \cap B c$, iff the $B$-modules $B / B b$ and $B / B c$ are isomorphic. 
Fix a faithfully flat extension $\Delta \rightarrow \prod_{i=1}^{s} \Delta_{c_{i}}$ as in Lemma 2.1 , where $\Delta_{i}=$ $\Delta_{c_{i}}=\Delta\left(D_{c_{i}}\right)$ is the localization of $\Delta$ at the powers of $c_{i} \in D$. Let $B=D_{*}^{-1} \Delta$ be the localization of $\Delta$ at $D_{*}=D \backslash\{0\}$. Then

$$
\hat{\Delta}=\hat{\Delta}(D \text {-torsion }) \cup \hat{\Delta}(D \text {-torsionfree }) .
$$

The ring $\Delta_{i}$ is the Ore extension $D_{c_{i}}\left[X_{i} ; \delta_{i}\right]$, it can be considered as the subring of $B$. Moreover, $B=B_{i}:=\left(D_{c_{i}}\right)_{*}^{-1} \Delta_{i}=l\left[X_{i} ; \delta_{i}\right]$ is the localization of $\Delta_{i}$ at $\left(D_{c_{i}}\right)_{*}=D_{c_{i}} \backslash\{0\}$. Then $X_{i}=\alpha_{i j} X_{j}+\beta_{i j}$ for some $0 \neq \alpha_{i j}, \beta_{i j} \in l$.

The ring $D$ is a commutative domain of Krull dimension 1 , thus, for $0 \neq c \in D$, the set $V(c)$ of prime ideals in $D$ containing $c$ is finite. Clearly, for $\mathbf{p} \in \operatorname{Specm} D$, the $\Delta$-module $\Delta / \Delta \mathrm{p}$ is holonomic (since $\Delta$ is a simple domain of Gelfand-Kirillov dimension 2 and in a view of Lemma 2.2 and (2.3)), hence, of finite length. Moreover,

$$
\Delta / \Delta \mathbf{p}=\bigcup_{i=1}^{\infty} \text { ann } \mathbf{p}^{i}
$$

where ann $\mathbf{p}^{i}=\left\{u \in \Delta / \Delta \mathbf{p}: \mathbf{p}^{i} u=0\right\}$.

Lemma 3.3. Let $M$ be a nonzero $\Delta$-module satisfying the following property: if $N$ is a nonzero submodule of $M$, then $N_{c_{i}} \neq 0$ for every $i$ such that $M_{c_{i}} \neq 0$ (e.g., $M$ is D-torsionfree; $M$ is an epimorphic image of $\Delta / \Delta \mathbf{p}$ for some $\mathbf{p} \in$ Specm $D)$. Then the $\Delta$-module $M$ is simple iff for each $i$ either $M_{c_{i}}=0$ or $M_{c_{i}}$ is a nonzero simple $\Delta_{i}$-module.

Proof. Evident.

Theorem 3.4. The map

$$
\text { Specm } D \rightarrow \hat{\Delta}(D \text {-torsion }), \mathbf{p} \rightarrow\left[\Delta / \Delta \mathbf{p} \simeq \Delta \otimes_{D} D / \mathbf{p}\right],
$$

is a bijection.

Proof. For a commutative Noetherian domain of Krull dimension 1 the restricted minimum condition holds (i.e., every proper factor ring is Artinian). Thus a simple $D$-torsion $\Delta$-module is an epimorphic image of the $\Delta$-module $\Delta / \Delta \mathbf{p}$ for some prime $\mathbf{p}$ of $D$. If $\mathbf{p}_{c_{i}} \neq D_{c_{i}}$, then it is the maximal ideal of $D_{c_{i}}$. The localization $(\Delta / \Delta \mathbf{p})_{c_{i}} \simeq \Delta_{i} / \Delta_{i} \mathbf{p}_{c_{i}}$ is either 0 or a simple $\Delta_{i}$-module (by Lemma 3.1 ). Hence, by Lemma 3.3 , every $\Delta$-module $\Delta / \Delta \mathbf{p}$ is simple. It means that a simple $D$-torsion $\Delta$-module is isomorphic to some $\Delta / \Delta \mathbf{p}$.

Since $\Delta / \Delta \mathbf{p}=\bigcup_{i=1}^{\infty}$ ann $\mathbf{p}^{i}$, where ann $\mathbf{p}^{i}=\left\{u \in \Delta / \Delta \mathbf{p}: \mathbf{p}^{i} u=0\right\}$, the $\Delta$-modules $\Delta / \Delta \mathbf{p}$ and $\Delta / \Delta \mathbf{p}^{\prime}$ are isomorphic iff $\mathbf{p}=\mathbf{p}^{\prime}$ ( $\mathbf{p}$ and $\mathbf{p}^{\prime}$ are primes).

Evidently, Specm $D_{c_{i}} \subseteq \operatorname{Specm} D$ and Specm $D=\bigcup_{i=1}^{s} \operatorname{Specm} D_{c_{i}}$. For every $i=1, \ldots, s$ we have the situation

$$
\Delta_{i}=D_{c_{i}}\left[X_{i} ; \delta_{i}\right] \rightarrow B_{i}=\left(D_{c_{i}}\right)_{*}^{-1} \Delta_{i}=l\left[X_{i} ; \delta_{i}\right]=B
$$

as at the beginning of this section.

Definition. We say that an element $b \in B$ is preserving with respect to the faithfully flat extension $\Delta \rightarrow \prod_{i=1}^{s} \Delta_{i}$ (or ext-preserving for short) if for every $i=1, \ldots, s$ the element $b \in B_{i}=B$ is preserving in the case $\Delta_{i} \rightarrow B_{i}=B$. 
Lemma 3.5. Suppose $0 \neq d_{1}, \ldots, d_{j} \in B$. Then there exists $0 \neq \xi \in D$ such that all elements $d_{1} \xi^{-1}, \ldots, d_{j} \xi^{-1}$ are preserving with respect to the faithfully flat extension $\Delta \rightarrow \prod_{i=1}^{s} \Delta_{i}$. More precisely, let $\left\{\mathbf{p}_{1}, \ldots, \mathbf{p}_{k}\right\}$ be those maximal ideals which are a special prime of at least one of $d_{1}, \ldots, d_{j}$ in at least one of the situations $\Delta_{i} \rightarrow B_{i}=B, i=1, \ldots, j$. Let $g_{l} \in \mathbf{p}_{l} \backslash \mathbf{p}_{l}^{2}(l=1, \ldots, k)$. Also take a natural number $v$ with $v \geq v_{l, i}$ for every $v_{l, i} \in \mathbf{N}$ (if any) such that $-v_{l, i}$ is a root of some $Q_{\mathbf{p}_{l}, d_{m}}^{(i)}$ in the case $\Delta_{i} \rightarrow B_{i}=B$. Then all elements $d_{m}\left(g_{1} \cdots g_{k}\right)^{-v}(m=1, \ldots, j)$ are preserving with respect to the faithfully flat extension $\Delta \rightarrow \prod_{i=1}^{s} \Delta_{i}$.

Proof. It follows immediately from Lemma 3.4, [B13].

Theorem 3.6. Let $b$ be an irreducible preserving with respect to the faithfully flat extension $\Delta \rightarrow \prod_{i=1}^{s} \Delta_{i}$ element of $B$. Then $M_{b}:=\Delta / \Delta \cap B b$ is a simple $D$ torsionfree $\Delta$-module. Up to isomorphism every simple $D$-torsionfree $\Delta$-module arises in this way and two such $\Delta$-modules are isomorphic, $M_{b} \simeq M_{c}$, iff the simple $B$-modules $B / B b$ and $B / B c$ are isomorphic.

Proof. Let $M$ be a simple $D$-torsionfree $\Delta$-module. Then the localization $D_{*}^{-1} M$ of $M$ at $D_{*}$ is a simple $B$-module, thus the module $D_{*}^{-1} M$ is isomorphic to the $B$-module $B / B b$ for some irreducible element $b$ of $B$. The $B$-modules $B / B b$ and $B / B b s^{-1}$ are isomorphic for every $s \in D_{*}$. By Lemma 3.5 we can suppose $b$ to be preserving (with respect to $\Delta \rightarrow \prod_{i=1}^{s} \Delta_{i}$ ). The $M$ is the $\Delta$-submodule of its localization $D_{*}^{-1} M \simeq B / B b$ (via $M \rightarrow D_{*}^{-1} M, m \rightarrow m / 1$ ). Moreover, $M=\operatorname{Soc}_{\Delta} B / B b$. Every nonzero $\Delta$-submodule of $D_{*}^{-1} M$ is essential, so $M \subseteq M_{b}$ and $0 \neq M_{c_{i}} \subseteq\left(M_{b}\right)_{c_{i}}$ for every $i$. The $\Delta_{i}$-modules $M_{c_{i}}$ and $\left(M_{b}\right)_{c_{i}}$ are simple (Theorem 3.2), hence $M_{c_{i}}=\left(M_{b}\right)_{c_{i}}$ for $i=1, \ldots, s$. The extension $\Delta \rightarrow \prod_{i=1}^{s} \Delta_{i}$ is faithfully flat, so $M=M_{b}$.

Let $b$ be as in the theorem. Then each $\left(M_{b}\right)_{c_{i}} \simeq \Delta_{i} / \Delta_{i} \cap B b$ is a nonzero simple $D_{c_{i}}$-torsionfree $\Delta_{i}$-module (by Theorem 3.2). By Lemma 3.3, the $\Delta$-module $M_{b}$ is simple. The other claims are evident.

\section{Simple holonomic modules}

Let $K$ be an algebraically closed field of characteristic zero and let the algebra

$$
\Lambda=C \otimes A
$$

be the tensor product of rings of differential operators with coefficients from a regular commutative affine domain of Krull dimension 1: $C=\Delta\left(D_{1}\right)$ and $A=$ $\Delta\left(D_{2}\right)$. Moreover, let

$$
A=D[X ; \delta], D=D_{2},
$$

be an Ore extension. Observe, that $\Lambda$ is isomorphic to the ring of differential operators $\Delta\left(D_{1} \otimes D_{2}\right)$ (Lemma 2.5).

Example. The second Weyl algebra $A_{2}=A_{1} \otimes A_{1}$ is an example of the ring $\Lambda$ $\left(A_{1}=K[t][X ; d / d t]\right)$.

Denote by $k$ the full quotient ring of $C$ :

$$
k=C_{*}^{-1} C, C_{*}=C \backslash\{0\},
$$

Then $k$ is a skew field (division ring) with center $Z(k)=K$. The ring

$$
\mathcal{A}:=C_{*}^{-1} \Lambda=k \otimes A=k \otimes D[X ; \delta]=\mathcal{D}[X ; \delta], \mathcal{D}=k \otimes D,
$$


is a ring of differential operators with $\operatorname{Ker} \delta \supseteq k$. The $\operatorname{ring} \mathcal{A}=\bigcup_{i \geq 0} \mathcal{A}_{i}$ is filtered by the powers of $X: \mathcal{A}_{i}=\bigoplus_{j \leq i} \mathcal{D} X^{j}=\bigoplus_{j \leq i} X^{j} \mathcal{D}$. The associated graded ring gr $\mathcal{A}=\bigoplus \mathcal{A}_{i} / \mathcal{A}_{i-1}$ is the polynomial ring $\mathcal{\mathcal { D }}[x]$ with coefficients from $\mathcal{D}$ where $x=X+\mathcal{D} \in \mathcal{A}_{1} / \mathcal{A}_{0}$.

Let $k$ be a skew field. We say that a ring $T$ is an affine algebra over $k$ if it is a factor ring of a polynomial ring $k\left[X_{1}, \ldots, X_{n}\right]$ with coefficients from $k$. In that case $T$ is generated over $k$ by the images $x_{i}$ of $X_{i}, T=k\left\langle x_{1}, \ldots, x_{n}\right\rangle$. The $\operatorname{ring} T$ is Noetherian and equipped with a standard filtration $\mathcal{T}=\left\{T_{i}, i \geq 0\right\}$ by the degree of the generators $T_{0}=k, T_{1}=k+\sum_{i=1}^{n} k x_{i}, T_{m}=\left(T_{1}\right)^{m}=\sum_{s \leq m} k x_{i_{1}} \cdots x_{i_{s}}$,

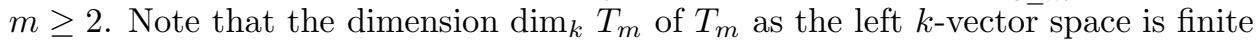
for every $m$. The associated graded ring $\operatorname{gr}_{\mathcal{T}} T=\bigoplus T_{i} / T_{i-1}$ is an affine $k$-algebra with generators of graded degree 1 (i.e. from $R_{1} / R_{0}$ ). Thus $\operatorname{gr}_{\mathcal{T}}$ is a Noetherian ring.

A filtration $\Gamma=\left\{\Gamma_{i}\right\}$ on a $T$-module $M\left(M=\bigcup \Gamma_{i}, T_{i} \Gamma_{j} \subseteq \Gamma_{i+j}\right)$ is called good if the associated graded $\operatorname{gr}_{\mathcal{T}} T$-module $\operatorname{gr}_{\Gamma} M=\bigoplus \Gamma_{i} / \Gamma_{i-1}$ is finitely generated. A $T$-module $M$ has a good filtration iff it is finitely generated (Noetherian). If $\left\{\Gamma_{i}\right\}$ and $\left\{\Omega_{i}\right\}$ are two good filtrations on $M$, then there exists a natural number $s$ such that $\Gamma_{i} \subseteq \Omega_{i+s}$ and $\Omega_{i} \subseteq \Gamma_{i+s}$ for all $i$. Let $M$ be a finitely generated $T$-module and let $M_{0}$ be a finitely generated $k$-module of generators of $M$. The filtration $\Gamma_{i}=\left\{T_{i} M_{0}, i \geq 0\right\}$ is called standard, if $\operatorname{dim}_{k} \Gamma_{i}<\infty$ for all $i \geq 0$. Every standard filtration is good.

A function $f: \mathbf{N} \rightarrow \mathbf{N}$ has polynomial growth if, for some $d \in \mathbf{R}, f(n) \leq n^{d}$ for $n>>0$; and then

$$
\gamma(f):=\inf \left\{d \in \mathbf{R}: f(n) \leq n^{d} \text { for sufficiently large } n>>0\right\}
$$

is called the degree of $f$.

Let $T=\bigcup_{i=0}^{\infty} T_{i}$ be as above and $M$ be a finitely generated $T$-module with a standard filtration $\Gamma=\left\{\Gamma_{i}\right\}$. The Gelfand-Kirillov dimension (with respect to the base division ring $k)$ : $\operatorname{GK}_{k}(T)=\gamma\left(\operatorname{dim}_{k} T_{i}\right)$ and $\operatorname{GK}_{k}(M)=\gamma\left(\operatorname{dim}_{k} M_{i}\right)$. The number $\mathrm{GK}_{k}(T)$ and $\mathrm{GK}_{k}(M)$ does not depend on the choice of standard filtration $\mathcal{T}$ and $\Gamma$. In case the division ring is the ground field $K$ we write GK for $\mathrm{GK}_{K}$.

Let $M$ be a $T$-module with a good filtration $\Gamma=\left\{\Gamma_{i}\right\}$. The integer valued function $\chi_{M, \Gamma}(i)=\operatorname{dim}_{k} \Gamma_{i}$ is called the Hilbert function of the module $M$ with respect to $\Gamma$. Standard arguments show that there exists a polynomial $H(t)=$ $H_{M, \Gamma}(t)=a_{d} t^{d}+\cdots+a_{0} \in \mathbf{Q}[t]$ with rational coefficients (see, for example, [Bav4] and Remark 2.5 there):

$$
\chi_{M, \Gamma}(i)=H_{M, \Gamma}(i) \text { for all } i>>0 .
$$

The polynomial $H_{M, \Gamma}$ is called the Hilbert polynomial of $M$ with respect to the filtration $\Gamma$. The degree $d=d(M)$ of $H_{M, \Gamma}$ coincides with $\operatorname{GK}_{k}(M)$. The positive integer $e(M)=d ! a_{d}$ is called the multiplicity of $M$. The degree $d(M)$ and the multiplicity $e(M)$ of $M$ do not depend on the choice of good filtration $\Gamma$.

Let $0 \rightarrow N \rightarrow M \rightarrow L \rightarrow 0$ be an exact sequence of finitely generated $T$-modules. Then

$$
\operatorname{GK}_{k}(M)=\max \left\{\operatorname{GK}_{k}(N), \operatorname{GK}_{k}(L)\right\}
$$

and if $\operatorname{GK}_{k}(N)=\operatorname{GK}_{k}(M)=\operatorname{GK}_{k}(L)$, then

$$
e(M)=e(N)+e(L) \text {. }
$$


In fact, let $\Gamma$ be a good filtration on $M$. Then $\Gamma_{i}^{\prime}=\left\{\Gamma_{i} \cap N\right\}$ and $\left\{\Gamma_{i}^{\prime \prime}=\left(\Gamma_{i}+N\right) / N\right\}$ are filtrations on $N$ and $L$ respectively, such that the sequence of graded $\operatorname{gr}_{\mathcal{T}} T$ modules

$$
0 \rightarrow \operatorname{gr}_{\Gamma^{\prime}} N \rightarrow \operatorname{gr}_{\Gamma} M \rightarrow \operatorname{gr}_{\Gamma^{\prime \prime}} L \rightarrow 0
$$

is exact. The ring $\operatorname{gr}_{\mathcal{T}} T$ is Noetherian and the $\operatorname{gr}_{\mathcal{T}} T$-module $\operatorname{gr}_{\Gamma} M$ is finitely generated, hence, Noetherian, thus the $\operatorname{gr}_{\mathcal{T}} T$-modules $\operatorname{gr}_{\Gamma^{\prime}} N$ and $\operatorname{gr}_{\Gamma^{\prime \prime}} L$ are finitely generated. Consequently, the filtrations $\Gamma^{\prime}$ and $\Gamma^{\prime \prime}$ are good and

$$
\chi_{M, \Gamma}(i)=\chi_{N, \Gamma^{\prime}}(i)+\chi_{L, \Gamma^{\prime \prime}}(i),
$$

hence,

$$
H_{M, \Gamma}(i)=H_{N, \Gamma^{\prime}}(i)+H_{L, \Gamma^{\prime \prime}}(i)
$$

and (4.1), (4.2) follow.

A non-left (resp. non-right, resp. non- ) Artinian ring satisfies the left (resp. right) restricted minimum condition (l.r.m.c., resp. r.r.m.c., resp. r.m.c.) provided every proper left (resp. right, resp. left and right) factor module of it is Artinian. The ring $\mathcal{D}$ is the tensor product of the central simple algebra $k$ and the Noetherian algebra $D$, hence, the ring $\mathcal{D}$ is a Noetherian $k$-affine algebra. Moreover, $\mathcal{D}$ is a Noetherian domain as the localization of the Noetherian domain $C \otimes D$.

Lemma 4.1. 1. For the ring $\mathcal{D}$ the restricted minimum condition holds;

2. $\mathrm{GK}_{k} \mathcal{D}=1$;

3. any proper (left or right) factor module of $\mathcal{D}$ is finitely generated over $k$. In particular, every simple $\mathcal{D}$-module is finitely generated over $k$.

Proof. 2. The $K$-algebra $D$ is a finitely generated module over a polynomial ring $K[H]$, so $\mathcal{D}$ is a finitely generated module over $k \otimes K[H]=k[H]$. Hence,

$$
1=\mathrm{GK}_{k}(k[H]) \leq \mathrm{GK}_{k}(\mathcal{D}) \leq \mathrm{GK}_{k}(k[H])=1 .
$$

3. Let us prove the statement for left modules. Let $x$ be a nonzero element of $\mathcal{D}$. The left ideal $\mathcal{D} x$ is isomorphic to $\mathcal{D}$ as a left module. For the exact sequence of $\mathcal{D}$-modules: $0 \rightarrow \mathcal{D} x \rightarrow \mathcal{D} \rightarrow \mathcal{D} / \mathcal{D} x \rightarrow 0$, let $\Gamma, \Gamma^{\prime}$, and $\Gamma^{\prime \prime}$ be good filtrations from (4.4). The Hilbert polynomials of $\mathcal{D}$ and $\mathcal{D} x \simeq \mathcal{D}$ are equal to $a i+b$ and $a i+c$, respectively for some integers $a, b, c$. It follows from (4.5) that the Hilbert polynomial of $\mathcal{D} / \mathcal{D} x$ is constant, i.e. $\operatorname{dim}_{k} \mathcal{D} / \mathcal{D} x<\infty$.

$3 \Rightarrow 1$. Evident.

Lemma 4.2. 1. Any proper factor module of ${ }_{\Lambda} \Lambda$ has Gelfand-Kirillov dimension $\leq 3$;

2. any nonzero submodule of $\Lambda \Lambda$ has Gelfand-Kirillov dimension 4;

3. for the algebra $C=\Delta\left(D_{1}\right)$ the restricted minimum condition holds and every simple C-module has Gelfand-Kirillov dimension 1.

Proof. Let $I$ be a nonzero left ideal of $\Lambda$ (resp. $C$ ) and let $0 \neq x \in I$. The $\Lambda$ (resp. $C)$ is the domain, so $\Lambda x \simeq \Lambda$ and $4=\mathrm{GK} \Lambda x \leq \mathrm{GK} I \leq \mathrm{GK} \Lambda=4$, i.e. GK $I=4$. By $[\mathrm{MR}, 8.3 .5, \operatorname{GK}(\Lambda / \Lambda x)<\mathrm{GK} \Lambda=4($ resp. $\operatorname{GK}(C / C x)<\mathrm{GK} C=2)$, hence $\operatorname{GK}(\Lambda / I) \leq \operatorname{GK}(\Lambda / \Lambda x) \leq 3$ (resp. $\operatorname{GK}(C / I) \leq \mathrm{GK}(C / C x) \leq 1$, i.e. any proper factor module of ${ }_{C} C$ is holonomic). 
Let $M$ be a nonzero simple $\Lambda$-module, the localization

$$
C_{*}^{-1} M=\mathcal{A} \otimes_{\Lambda} M
$$

of the module $M$ at $C_{*}$ is either zero or not. The latter is a simple $\mathcal{A}$-module. With respect to these two possibilities we say that the module $M$ is either $C$-torsion or $C$-torsionfree, so

$$
\hat{\Lambda}=\hat{\Lambda}(C \text {-torsion }) \cup \hat{\Lambda}(C \text {-torsionfree }) .
$$

Let $R$ be a $K$-algebra and let $M$ be a simple $R$-module such that the endomorphism ring $\operatorname{End}_{R}(M)=K$. Let $S$ be a $K$-algebra and $N$ be an $S$-module. Then the tensor product $M \otimes N$ is an $R \otimes S$-module. By [Bav5], any submodule of $M \otimes N$ is equal to $M \otimes N^{\prime}$ for some $S$-submodule $N^{\prime}$ of $N$. In particular, $M \otimes N$ is a simple $R \otimes S$-module if ${ }_{S} N$ is simple, i.e.

$$
\hat{R} \otimes \hat{S} \subseteq(R \otimes S)^{\wedge}
$$

$$
\hat{\Delta}\left(D_{1}\right) \otimes \hat{\Delta}\left(D_{2}\right) \subseteq \hat{\Delta}\left(D_{1} \otimes D_{2}\right)
$$

Proposition 4.3.

$$
\hat{\Lambda}(C \text {-torsion })=\hat{C} \otimes \hat{A} \subseteq \hat{\Lambda}(\text { holonomic }) .
$$

Proof. The inclusion $\hat{C} \otimes \hat{A} \subseteq \hat{\Lambda}$ (holonomic) follows from (4.7) and Lemma 4.2.(3). The inclusion $\hat{\Lambda}(C$-torsion $) \supseteq \hat{C} \otimes \hat{A}$ is evident (Lemma 4.2.(3)).

Let $M$ be a nonzero simple $C$-torsion $\Lambda$-module. The algebra $C$ satisfies the restricted minimum condition, so $M$ contains a simple $C$-submodule, say $N$. Then the $\Lambda$-module $M$ is an epimorphic image of the $\Lambda$-module $N \otimes A$. Hence, $M \simeq$ $N \otimes A / J$ for some maximal left ideal $J$ of $A$, i.e. $\hat{\Lambda}(C$-torsion $) \subseteq \hat{C} \otimes \hat{A}$.

Denote by $h$ the skew field $\mathcal{D}_{*}^{-1} \mathcal{D}$ where $\mathcal{D}_{*}=\mathcal{D} \backslash\{0\}$ and by

$$
\mathcal{B}:=\mathcal{D}_{*}^{-1} \mathcal{A}=h[X ; \delta]
$$

the localization of the algebra $\mathcal{A}$ at $\mathcal{D}_{*}$. Observe that $h$ is the full quotient ring of the Noetherian domain $C \otimes D$.

We identify $\mathcal{A}$ with its image in $\mathcal{B}$ via the algebra monomorphism $\mathcal{A} \rightarrow \mathcal{B}$, $x \rightarrow x / 1$. So, we have

$$
\hat{\mathcal{A}}=\hat{\mathcal{A}}(\mathcal{D} \text {-torsion }) \cup \hat{\mathcal{A}}(\mathcal{D} \text {-torsionfree }) .
$$

Lemma 4.4. If $[N] \in \hat{\mathcal{A}}(\mathcal{D}$-torsionfree, $\Lambda$-socle $)$, then GK Soc $_{\Lambda} N=3$.

Proof. Since $\operatorname{Soc}_{\Lambda} N \neq 0$ and $N$ is $D$-torsionfree, the socle $\operatorname{Soc}_{\Lambda} N$ is a simple $\Lambda$-module that contains a free $C \otimes D$-module. Hence $4=\operatorname{GK} \Lambda>\operatorname{GK}\left(\operatorname{Soc}_{\Lambda} N\right)$ and

$$
3 \geq \operatorname{GK}\left(\operatorname{Soc}_{\Lambda} N\right) \geq \operatorname{GK}(C \otimes D)=\operatorname{GK} C+\operatorname{GK} D=2+1=3 .
$$

Let $V=\mathcal{D} / I$ be a simple $\mathcal{D}$-module for some maximal left ideal $I$ of $\mathcal{D}$. The endomorphism ring

$$
\varepsilon \equiv \varepsilon(V) \equiv \operatorname{End}_{\mathcal{D}}(V)
$$


is a skew field and $V={ }_{\mathcal{D}} V_{\varepsilon}$ is a $(\mathcal{D}, \varepsilon)$-bimodule (in this situation we write endomorphisms from $\varepsilon$ on the right). The induced $(\mathcal{A}, \varepsilon)$-bimodule

$$
\mathcal{A}(V):=\mathcal{A} \otimes_{\mathcal{D}} V=\bigoplus_{i \geq 0} X^{i} \otimes V
$$

is a filtered $\mathcal{A}$-module

$$
\mathcal{A}(V)=\bigcup_{i \geq 0} \mathcal{A}(V)_{i}, \quad \mathcal{A}(V)_{i}=\bigoplus_{j \leq i} X^{j} \otimes V .
$$

Note that every $\mathcal{A}(V)_{i}$ is a $(\mathcal{D}, \varepsilon)$-bimodule. A nonzero element $u \in \mathcal{A}(V)$ can be uniquely written as a sum

$$
u=1 \otimes u_{0}+X \otimes u_{1}+\cdots+X^{n} \otimes u_{n}, u_{i} \in V, u_{n} \neq 0 .
$$

The number $n:=\operatorname{deg} u$ is called the degree of $u$. Clearly, $u \in \mathcal{A}(V) \operatorname{deg} u \backslash \mathcal{A}(V)_{\operatorname{deg} u-1}$ and $\operatorname{deg} u$ is defined by this property. The elements $X^{n} \otimes u_{n}$ and $u_{n}$ are called the leading term and the leading coefficient of $u$ respectively. An element from $\mathcal{A}(V)$ is called monic if its leading coefficient is $\overline{1}:=1+I$.

The right $\mathcal{D}$-module $\mathcal{A}$ is free, so applying the exact functor $\mathcal{A} \otimes_{\mathcal{D}}$ - to the exact sequence of $\mathcal{D}$-modules: $0 \rightarrow I \rightarrow \mathcal{D} \rightarrow V \rightarrow 0$ we obtain the canonical isomorphism of $\mathcal{A}$-modules:

$$
\mathcal{A} / \mathcal{A} I \rightarrow \mathcal{A}(V), \quad \sum X^{i} d_{i}+\mathcal{A} I \rightarrow \sum X^{i} \otimes\left(d_{i}+I\right), \quad d_{i} \in \mathcal{D} .
$$

We identify the $\mathcal{A}$-modules $\mathcal{A} / \mathcal{A} I$ and $\mathcal{A}(V)$ via (4.11).

Lemma 4.5. Let $[M] \in \hat{\mathcal{A}}(\mathcal{D}$-torsion). Then $M$ is an epimorphic image of the $\mathcal{A}$-module $\mathcal{A}(V)$ for some $[V] \in \hat{\mathcal{D}}$.

Proof. Follows immediately from Lemma 4.1.(1).

Lemma 4.6. Suppose $\mathcal{A}(V)$ is a simple $\Lambda$-socle $\mathcal{A}$-module for some $[V] \in \hat{\mathcal{D}}$. Then $\mathrm{GK} \mathrm{Soc}_{\Lambda} \mathcal{A}(V)=3$, i.e. it is a simple non-holonomic $\Lambda$-module.

Proof. Observe that $\operatorname{Soc}_{\Lambda} \mathcal{A}(V)$ is a simple $\Lambda$-module and, for any nonzero $u \in$ $\mathcal{A}(V)$, the $C \otimes K[X]$-submodule $C \otimes K[X] u$ of $\mathcal{A}(V)$ is free. Choose $u$ from $\operatorname{Soc}_{\Lambda} \mathcal{A}(V)$. Then $3 \geq \operatorname{GKSoc}_{\Lambda} \mathcal{A}(V) \geq \mathrm{GK}(C \otimes K[X] C \otimes K[X] u)=\mathrm{GK} C \otimes K[X]=$ $\mathrm{GK} C+\mathrm{GK} K[X]=2+1=3$, i.e. $\mathrm{GK} \mathrm{Soc}_{\Lambda} \mathcal{A}(V)=3$.

Let $R$ be a ring and let $J$ be a left ideal of $R$. The $\operatorname{ring} \mathcal{I}(J)=\{r \in R \mid J r \subseteq J\}$ is called the idealizer of $J$, and is easily seen to be the largest subring of $R$ containing $J$ as an ideal. The ring $\overline{\mathcal{I}}(J)=\mathcal{I}(J) / J$ is called the eigenring of $J$. This acts, by right multiplication, on the module $R / J$ and it can be checked that $\overline{\mathcal{I}}(J)$ is canonically isomorphic to the endomorphism $\operatorname{ring} \operatorname{End}_{R}(R / J)$ of the $R$-module $R / J: \quad\left(v \leftrightarrow\left(f_{v}: 1+J \rightarrow v\right)\right)$. In this case we write endomorphisms from $\operatorname{End}_{R}(R / J)$ on the right, i.e. $(u) f_{v}=u v$. We identify $\overline{\mathcal{I}}(J)$ and $\operatorname{End}_{R}(R / J)$ via the isomorphism above. We have

$$
\begin{gathered}
\operatorname{End}_{\mathcal{D}}(\mathcal{D} / I) \equiv \overline{\mathcal{I}}(I)=\operatorname{ann}_{\mathcal{D} / I}(I) \subseteq \mathcal{D} / I \\
\operatorname{End}_{\mathcal{A}}(\mathcal{A} / \mathcal{A} I) \equiv \overline{\mathcal{I}}(\mathcal{A} I)=\operatorname{ann} \mathcal{A} / \mathcal{A} I(I) \subseteq \mathcal{A} / \mathcal{A} I \equiv \mathcal{A}(V),
\end{gathered}
$$

where ann ${ }_{\mathcal{D} / I}(I)=\{u \in \mathcal{D} / I: I u=0\}$, etc. Set $\mathcal{E}=\mathcal{E}(\mathcal{A} / \mathcal{A} I)$ for the endomorphism ring $\operatorname{End}_{\mathcal{A}}(\mathcal{A} / \mathcal{A} I)$. The ring $\mathcal{E}$ is a filtered ring, $\mathcal{E}=\bigcup_{i \geq 0} \mathcal{E}_{i}$, where $\mathcal{E}_{i}=\mathcal{E} \cap \mathcal{A}(V)_{i}\left(\mathcal{E}_{i} \mathcal{E}_{j} \subseteq \mathcal{E}_{i+j}\right.$ for all $\left.i, j \geq 0\right)$. Clearly, $\mathcal{E}_{0}=\varepsilon$. 
Define $H^{0}=\left\{i: \mathcal{E}_{i-1} \neq \mathcal{E}_{i}\right\}$. Then $0 \in H^{0} \quad(I \cdot 1 \otimes \overline{1}=0)$. Let $u_{i}=$ $\sum_{j=0}^{i} X^{j} \otimes \lambda_{i j} \in \mathcal{E}_{i} \backslash \mathcal{E}_{i-1}\left(\lambda_{i j} \in V, \lambda_{i i} \neq 0\right)$; then

$$
0=I u_{i}=\sum_{k=0}^{i} X^{k} \otimes \sum_{k \leq j}^{i}(-1)^{j-k}\left(\begin{array}{l}
j \\
k
\end{array}\right) \delta^{j-k}(I) \lambda_{i j},
$$

or, equivalently,

$$
\sum_{k \leq j}^{i}(-1)^{j-k}\left(\begin{array}{l}
j \\
k
\end{array}\right) \delta^{j-k}(I) \lambda_{i j}=0, \quad k=0, \ldots, i .
$$

If $k=i$, then $I \lambda_{i i}=0$, so $0 \neq \lambda_{i i} \in \varepsilon$. Therefore, for every $i \in H^{0}$ there exists

$$
u_{i}=\sum_{j=0}^{i} X^{j} \otimes \lambda_{i j} \in \mathcal{E}_{i} \backslash \mathcal{E}_{i-1} \text { with } \lambda_{i i}=\overline{1}=1+I .
$$

The set $\left\{u_{i}, i \in H^{0}\right\}$ from (4.15) is a left and right $\varepsilon$-basis of $\mathcal{E}$ :

$$
\mathcal{E}=\bigoplus_{i \in H^{0}} \varepsilon u_{i}=\bigoplus_{i \in H^{0}} u_{i} \varepsilon, \quad \mathcal{E}_{i}=\bigoplus_{j \leq i} \varepsilon u_{j}=\bigoplus_{j \leq i} u_{j} \varepsilon
$$

By (4.15), $\mathcal{E}=\bigoplus_{j \leq i} \mathcal{E}_{i}$ is an $H^{0}$-filtered domain and $H^{0}$ is an additive submonoid of $\mathbf{N}_{0}$.

Let $i \in H^{0}$ and let $j$ be the maximal positive element of $H^{0}$ with $j<i$. For $\lambda \in \varepsilon, \lambda u_{i}-u_{i} \lambda \in \mathcal{E}_{j}$. Suppose $H^{0} \neq 0$. Let $m$ be the minimal positive element of $H^{0}$ and let $g>0$ be the greatest common divisor of the elements of $H^{0}$. Evidently, $\mathbf{Z} \geq \mathbf{Z} H^{0}=g \mathbf{Z}$. Choose $0<i, j \in H^{0}$ such that $g=j-i$. All elements

$$
i^{2}+(k+l i) g=l g i+(i-k) i+k j, \quad k=0, \ldots, i-1, l \geq 0,
$$

belong to $H^{0}$, thus the following definition is correct. The starting point $\operatorname{sp}\left(H^{0}\right)$ of $H^{0}$ is the minimal nonzero $h \in H^{0}$ such that $i g \in H^{0}$ for all $i \geq h g^{-1}$. Thus for any $k \in H^{0}$ the set $H^{0} \backslash\left\{k+H^{0}\right\}$ is finite. The existence of the starting point guarantees that for each $j=0, \ldots, g^{-1} m-1$ there exists a minimal element $m_{j}$ of $H^{0}$ with $m_{j} \equiv j g(\bmod m)$. We have a partition

$$
H^{0}=\bigcup_{j=0}^{g^{-1} m-1}\left(m_{j}+m \mathbf{N}_{0}\right)
$$

The subring $R$ of $\mathcal{E}$ generated by $\varepsilon$ and $u_{m}$ is the differential operator ring

$$
R=\varepsilon\left[u_{m} ; \partial\right], \quad \partial \lambda=u_{m} \lambda-\lambda u_{m}, \quad \lambda \in \varepsilon .
$$

By (4.15),

$$
\partial \bar{\mu}=\lambda_{m 0} \mu-\mu \lambda_{m 0}-\sum_{j=1}^{m}(-1)^{j} \delta^{j}(\mu) \lambda_{m j}+I, \quad \bar{\mu}=\mu+I \in \varepsilon .
$$

For $u, v \in \mathcal{E}, \operatorname{deg}(u+v) \leq \max \{\operatorname{deg} u, \operatorname{deg} v\}$ and $\operatorname{deg}(u v)=\operatorname{deg} u+\operatorname{deg} v$.

Lemma 4.7. Suppose that $H^{0} \neq 0$. Then

1. Each proper left (right) factor module of $\mathcal{E}$ is a finite dimensional left (right) $\varepsilon$-vector space, so the ring $\mathcal{E}$ satisfies the restricted minimum condition and is a Noetherian domain; 
2. the ring $\mathcal{E}$ is a finitely generated free left and right $R$-module

$$
\mathcal{E}=\bigoplus_{j=0}^{g^{-1} m-1} R u_{m_{j}}=\bigoplus_{j=0}^{g^{-1} m-1} u_{m_{j}} R
$$

Proof. Evident.

Denote by Mon $\mathcal{E}$ (respectively Irr.mon $\mathcal{E}$ ) the set of all (respectively monic and irreducible) elements of $\mathcal{E}$; and by $\operatorname{Submod} \mathcal{A}(V)($ resp. Max.submod $\mathcal{A}(V))$ the set of all (resp. maximal $A$-submodules) of $\mathcal{A}(V)$. Denote by $\operatorname{Sim}$.fac $\mathcal{A}(V)$ the set of isoclasses of simple epimorphic images of the $\mathcal{A}$-module $\mathcal{A}(V)$.

Proposition 4.8. Let $[V=\mathcal{D} / I] \in \hat{\mathcal{D}}$.

1. Any proper factor module (resp. submodule) of $\mathcal{A}(V)$ has finite (resp. infinite) length as a left $\mathcal{D}$-module;

2. any nonzero homomorphism of the $\mathcal{A}$-module $\mathcal{A}(V)$ is a monomorphism;

3. the map

$$
\operatorname{Mon} \mathcal{E} \rightarrow \operatorname{Submod} \mathcal{A}(V) \backslash\{0\}, \quad v \rightarrow \mathcal{A} v,
$$

is a bijection with inverse $0 \neq N \rightarrow\left\{0 \neq v=X^{i} \otimes \overline{1}+\cdots \in N\right.$ has minimal degree $i\}$, where $\overline{1}=1+I \in V$; and $\mathcal{A}(V)=\mathcal{A}(V)_{i-1} \oplus \mathcal{A} v$, where $i=\operatorname{deg} v$;

4. let $v, u \in \operatorname{Mon} \mathcal{E}$. Then $A v \subseteq A u$ iff $v=w u$ for some $w \in \operatorname{Mon} \mathcal{E}$. Hence, the map

$$
\text { Irr.mon } \mathcal{E} \rightarrow \operatorname{Max} . \operatorname{submod} \mathcal{A}(V), \quad v \rightarrow \mathcal{A} v,
$$

is a bijection;

5. any nonzero submodule of $\mathcal{A}(V)$ is isomorphic to $\mathcal{A}(V)$;

6. the $\mathcal{A}$-module $\mathcal{A}(V)$ is simple iff $\left(\operatorname{ann}{ }_{\mathcal{A}(V)} I \equiv \overline{\mathcal{I}}(\mathcal{A} I) \equiv\right) \mathcal{E}=\varepsilon$.

7. The map

$$
\text { Irr.mon } \mathcal{E} \rightarrow \operatorname{Sim} . \text { fac } \mathcal{A}(V), \quad v \rightarrow[\mathcal{A}(V) / \mathcal{A} v],
$$

is surjective; and the $\mathcal{D}$-length $l_{\mathcal{D}}(\mathcal{A}(V) / \mathcal{A} v)=\operatorname{deg} v<\infty$.

8. every nonzero $\Lambda$-submodule ( $\mathcal{A}$-submodule) of $\mathcal{A}(V)$ is essential, hence the $\mathcal{A}$-module $\mathcal{A}(V)$ is indecomposable;

9. if the $\mathcal{A}$-module $\mathcal{A}(V)$ is not simple, then $\operatorname{Soc}_{\Lambda} \mathcal{A}(V)=0$ and any nonzero finitely generated $\Lambda$-submodule of $\mathcal{A}(V)$ has Gelfand-Kirillov dimension 3;

10. for any $v \in \mathcal{E} \backslash \varepsilon: \bigcap_{i=1}^{\infty} \mathcal{A} v^{i}=0$.

Proof. 1. Let $0 \neq u \in \mathcal{A}(V)_{i} \backslash \mathcal{A}(V)_{i-1} \quad(i \geq 0)$. Then $\mathcal{A}(V)_{i-1}+\mathcal{A} u=\mathcal{A}(V)$ and the result follows $l_{\mathcal{D}}\left(\mathcal{A}(V) / \mathcal{A}(V)_{i-1}\right)=l_{\mathcal{D}} \mathcal{A}(V)_{i}=i+1<\infty$ and $l_{\mathcal{D}} \mathcal{A}(V)=\infty$, where $l_{\mathcal{D}} M$ is the length of a $\mathcal{D}$-module $M$.

$1 \Rightarrow 2$. Evident.

3. Let $N$ be a nonzero $\mathcal{A}$-submodule of $\mathcal{A}(V)$ and let $v=X^{i} \otimes \overline{1}+\cdots,(\overline{1}=$ $1+I \in V$ ) be a nonzero element of $N$ of minimal degree $i$. The element $v$ is uniquely defined. Then $v \in$ ann ${ }_{\mathcal{A}(V)} I \equiv \mathcal{E}$ and $N \cap \mathcal{A}(V)_{i-1}=0$. It follows from $\mathcal{A}(V)_{i-1}+\mathcal{A} v=\mathcal{A}(V)$ and $\mathcal{A} v \subseteq N$, that $\mathcal{A}(V)=\mathcal{A}(V)_{i-1} \oplus N=\mathcal{A}(V)_{i-1} \oplus \mathcal{A} v$, hence $N=\mathcal{A} v$, i.e. the map (4.19) is surjective. A monic element $v^{\prime}$ has minimal degree in the module $\mathcal{A} v^{\prime}$. By the uniqueness of $v^{\prime}$, the map (4.19) is injective.

4. Let $\mathcal{A} v \subseteq \mathcal{A} u$ for some nonzero $u, v \in \operatorname{Mon} \mathcal{E}$. Then $v$ and $u$ are nonzero elements of the submodules $\mathcal{A} v$ and $\mathcal{A} u$ of minimal degree. By 2 , the $\mathcal{A}$-module $\mathcal{A} u$ is isomorphic to $\mathcal{A}(V)$ and $\mathcal{A} v$ is a submodule of $\mathcal{A} u$. By the surjectivity of 
the map (4.19), $v=w u$ for some $w \in \mathcal{E}$. If $v=w u$, for some $w, u \in$ Mon $\mathcal{E}$, then $\mathcal{A} v \subseteq \mathcal{A} u$. If, in addition, $\operatorname{deg} w \geq 1$, then $\mathcal{A} v \subset \mathcal{A} u$. It follows that the map (4.19.1) is correctly defined and bijective, in view of (4.19).

$2 \Rightarrow 5 ; 3 \Rightarrow 6$; and $4 \Rightarrow 7$.

8. Suppose $M$ and $N$ are nonzero $\Lambda$-submodules of $\mathcal{A}(V)$ with $M \cap N=0$. Then $C_{*}^{-1} M$ and $C_{*}^{-1} N$ are nonzero $\mathcal{A}$-submodules of $\mathcal{A}(V)$ with $C_{*}^{-1} M \cap C_{*}^{-1} N=0$. The module $C_{*}^{-1} M$ can be considered as an $\mathcal{A}$-submodule of the factor module $\mathcal{A}(V) / C_{*}^{-1} N$. By 1 , the former has infinite $\mathcal{D}$-length but the latter has finite, a contradiction.

9. If $\operatorname{Soc}_{\Lambda} \mathcal{A}(V)$ is nonzero, then it is a simple $\Lambda$-module (since, by 8 , any nonzero $\Lambda$-submodule is essential) and the localization $C_{*}^{-1}\left(\operatorname{Soc}_{\Lambda} \mathcal{A}(V)\right)$ is the simple $\mathcal{A}$ submodule of $\mathcal{A}(V)$. By 5 , the $\mathcal{A}$-module $\mathcal{A}(V)$ is simple, a contradiction. Thus $\operatorname{Soc}_{\Lambda} \mathcal{A}(V)=0$.

By the canonical $\Lambda$-monomorphism

$$
L:=\Lambda / \Lambda \cap \mathcal{A} I \rightarrow \mathcal{A}(V) \equiv \mathcal{A} / \mathcal{A} I, u+\Lambda \cap \mathcal{A} I \rightarrow u+\mathcal{A} I, u \in \Lambda,
$$

we identify $L$ with its image in $\mathcal{A}(V)$. The left ideal $\Lambda \cap \mathcal{A} I$ is nonzero, since $C_{*}^{-1}(\Lambda \cap \mathcal{A} I)=\mathcal{A} I \neq 0$. The $\Lambda$-module $L$ is a proper factor module of $\Lambda$. It follows from Lemma 4.2.(1) that GK $L \leq 3$. Let $M$ be a nonzero cyclic $\Lambda$-submodule of $\mathcal{A}(V)$. By $8, N=M \cap L \neq 0$ and GK $N \leq 3$, hence the $\Lambda$-module $M$ is not isomorphic to ${ }_{\Lambda} \Lambda$ (Lemma 4.2(2)). Thus any nonzero finitely generated $\Lambda$ submodule $P$ of $\mathcal{A}(V)$ has Gelfand-Kirillov dimension $\leq 3$. If GK $P=2$ for some $P$, then $P$ contains a simple $\Lambda$-submodule, hence $\operatorname{Soc}_{\Lambda} \mathcal{A}(V) \neq 0$, a contradiction, i.e. GK $P=3$ for any nonzero finitely generated $\Lambda$-module $P$ of $\mathcal{A}(V)$.

10. Evident (degree argument).

Observe that every $k$-finite dimensional $\mathcal{A}$-module is $\mathcal{D}$-torsion. So,

$$
\hat{\mathcal{A}}(\mathcal{D} \text {-torsion })=\hat{\mathcal{A}}(k \text {-fin.dim }) \cup \hat{\mathcal{A}}(\mathcal{D} \text {-torsion, } k \text {-inf.dim }),
$$

where a simple $\mathcal{D}$-torsion $\mathcal{A}$-module belongs to the first, respectively the second set, if it is $k$-finite respectively $k$-infinite dimensional. By Proposition 4.8, Lemma 4.1.(3) and Lemma 4.6,

$$
\begin{aligned}
& \hat{\mathcal{A}}(\mathcal{D} \text {-torsion, } k \text {-inf.dim })=\left\{[\mathcal{A}(V)], \text { where }{ }_{\mathcal{A}} \mathcal{A}(V) \text { is simple }\right\}, \\
& \hat{\mathcal{A}}(k \text {-fin.dim })=\cup\left\{\operatorname{Sim} \text {.fac } \mathcal{A}(V), \text { where }{ }_{\mathcal{A}} \mathcal{A}(V) \text { is not simple }\right\},
\end{aligned}
$$

where $\operatorname{Sim} . \operatorname{fac} \mathcal{A}(V)$ is the set of isoclasses of simple epimorphic images of the $\mathcal{A}$ module $\mathcal{A}(V)$. A module $M$ is called GK-critical provided any proper factor module of $M$ has Gelfand-Kirillov dimension less than GK $M$.

Theorem 4.9. The map

$$
\hat{\Lambda}(\text { holonomic }) \backslash \hat{C} \otimes \hat{A} \rightarrow \hat{\mathcal{A}}(k-\text { fin.dim }),[M] \rightarrow\left[\mathcal{A} \otimes_{\Lambda} M\right],
$$

is bijective with inverse $[N] \rightarrow\left[\operatorname{Soc}_{\Lambda} N\right]$.

Proof. For $[M] \in \hat{\Lambda}$ (holonomic) $\backslash \hat{C} \otimes \hat{A}, \tilde{M}:=\mathcal{A} \otimes_{\Lambda} M$ is the (nonzero) simple $\mathcal{A}$ module with $\operatorname{Soc}_{\Lambda} \tilde{M}=M$. By Lemma 4.4, the $\mathcal{A}$-module $\tilde{M}$ is $\mathcal{D}$-torsion, hence, by Lemma $4.5, \tilde{M}$ is an epimorphic image of $\mathcal{A}(V)$ for some $[V] \in \hat{\mathcal{D}}$. By Lemma 4.6, $\tilde{M}$ is a proper epimorphic image of the non-simple $\mathcal{A}$-module $\mathcal{A}(V)$ and, by (4.23), $[\tilde{M}] \in \hat{\mathcal{A}}(k$-fin.dim). So, the map (4.24) is well-defined and injective, since $\operatorname{Soc}_{\Lambda} \tilde{M}=M$. 
It remains to be proved that for every non-simple $\mathcal{A}$-module $\mathcal{A}(V)$ the $\Lambda$-socle of every simple epimorphic image $N$ of $\mathcal{A}(V)$ has Gelfand-Kirillov dimension 2, i.e. is holonomic (then, evidently, $[N] \in \hat{\Lambda}$ (holonomic) $\backslash \hat{C} \otimes \hat{A}$ and $N=\mathcal{A} \otimes_{\Lambda} \operatorname{Soc}_{\Lambda} N$, i.e. the map $[N] \rightarrow\left[\operatorname{Soc}_{\Lambda} N\right]$ is inverse to $\left.(4.24)\right)$. Let $[V=\mathcal{D} / I] \in \hat{\mathcal{D}}$ for some maximal left ideal $I$ of $\mathcal{D}$. Let $N$ be a simple epimorphic image of the $\mathcal{A}$-module $\mathcal{A}(V) \equiv \mathcal{A} / \mathcal{A} I$. By Proposition 4.8.(7), $N=\mathcal{A}(V) / \mathcal{A} v$ for some irreducible monic element $v=v^{\prime}+\mathcal{A} I\left(v^{\prime} \in \mathcal{A}\right)$ of $\mathcal{E} \equiv \overline{\mathcal{I}}(\mathcal{A} I) \equiv \operatorname{ann}_{\mathcal{A}(V)} I \subseteq \mathcal{A}(V) \equiv \mathcal{A} / \mathcal{A} I$. By Proposition 4.8.(5), we have the $\mathcal{A}$-module isomorphism

$$
(\cdot) v: \mathcal{A}(V) \rightarrow \mathcal{A} v, \quad u \rightarrow u v .
$$

The algebra $\Lambda$ is somewhat commutative. Fix a finite dimensional filtration of the algebra $\Lambda=\bigoplus_{i \geq 0} \Lambda_{i}$ such that the associated graded algebra gr $\Lambda=\bigoplus_{i \geq 0} \Lambda_{i} / \Lambda_{i-1}$ is affine commutative. The $\mathcal{A}$-module $\mathcal{A}(V)$ is not simple, so any nonzero finitely generated $\Lambda$-submodule of $\mathcal{A}(V)$ has Gelfand-Kirillov dimension 3 (Proposition 4.8.(9)). Choose a nonzero finitely generated $\Lambda$-submodule, say $M$, of $\mathcal{A}(V)$ which has minimal possible multiplicity $e(M)$ (such a module exists because of Lemma 2.2 and additivity of multiplicity (2.3)). Then $M$ is GK-critical (if not, then there is a nonzero submodule $L$ of $M$ with $\operatorname{GK}(M / L)=3$. We have the exact sequence of $\Lambda$-modules: $0 \rightarrow L \rightarrow M \rightarrow M / L \rightarrow 0$ with $\operatorname{GK} L=\operatorname{GK} M=\operatorname{GK}(M / L)=3$, hence, by $(2.3), e(L)=e(M)-e(M / L)<e(M)$ which contradicts the choice of $M)$. By Proposition 4.8.(10) we have the descending chain of $\mathcal{A}$-submodules

$$
\mathcal{A}(V) \supset \mathcal{A} v \supset \cdots \supset \mathcal{A} v^{i} \supset \cdots, \text { with } \bigcap_{i=1}^{\infty} \mathcal{A} v^{i}=0
$$

and each factor $\mathcal{A} v^{i} / \mathcal{A} v^{i+1}$ is isomorphic to $N$ (since the map $v^{i}: \mathcal{A}(V) \rightarrow \mathcal{A} v$, $u \rightarrow u v^{i}$, is the $\mathcal{A}$-module isomorphism, Proposition 4.8). There exists $i \geq 0$ : $M \subseteq \mathcal{A} v^{i}$ and $M \nsubseteq \mathcal{A} v^{i+1}$. On the one hand, the nonzero finitely generated $\Lambda$ submodule $Q=M / M \cap \mathcal{A} v^{i+1}$ is a submodule of $\mathcal{A} v^{i} / \mathcal{A} v^{i+1} \simeq N$. On the other hand, $Q$ is a proper factor module of the critical $\Lambda$-module $M$, so GK $Q=2$. The $\Lambda$-module $Q$ is holonomic, hence, it contains a simple holonomic submodule, say $U$. Evidently, $U=\operatorname{Soc}_{\Lambda} N$.

Remark. In the proof of the last step of the theorem above we have not used the irreducibility of $v$. So, in fact, we have proved the following corollary.

Corollary 4.10. Suppose the $\mathcal{A}$-module $\mathcal{A}(V)$ is not simple for some $[V] \in \hat{\mathcal{D}}$. Then any proper factor module of $\mathcal{A}(V)$ contains a simple holonomic $\Lambda$-submodule from $\hat{\Lambda}$ (holonomic $) \backslash \hat{C} \otimes \hat{A}$.

Corollary 4.11. Let $M$ be a nonzero simple $\Lambda$-module and let $\tilde{M}=\mathcal{A} \otimes_{\Lambda} M$. Then

1. $\tilde{M}=0 \Leftrightarrow[M] \in \hat{C} \otimes \hat{A}$;

2. $1 \leq \operatorname{dim}_{k} \tilde{M}<\infty \Leftrightarrow[M] \in \hat{\Lambda}$ (holonomic $) \backslash \hat{C} \otimes \hat{A}$;

3. $\operatorname{dim}_{k} \tilde{M}=\infty \Leftrightarrow[M] \in \hat{\Lambda}($ non-holonomic $)$.

Hence, $M$ is holonomic (resp. non-holonomic) iff $\operatorname{dim}_{k} \tilde{M}<\infty$ (resp. $M$ contains a free $C \otimes K[X]$-module of rank 1$)$.

Proof. 1. It follows from Proposition 4.3.

2 and 3 follow from Theorem 4.9. 
Corollary 4.12. Let $[V=\mathcal{D} / I] \in \hat{\mathcal{D}}$ for a maximal left ideal $I$ of $\mathcal{D}$ such that the $\mathcal{A}$-module $\mathcal{A}(V) \equiv \mathcal{A} / \mathcal{A} I$ is not simple (i.e. the left ideal $\mathcal{A} I$ of $\mathcal{A}$ is not maximal) and let $v=\tilde{v}+\mathcal{A} I(\tilde{v} \in \mathcal{A})$ be an irreducible element of the ring $\mathcal{E}=\mathcal{E}(\mathcal{A} / \mathcal{A} I)$. Then

1. the $\Lambda$-module

$$
M(I, \tilde{v}):=\Lambda / \Lambda \cap \mathcal{A}(I, \tilde{v})
$$

is a $\Lambda$-submodule of $\mathcal{A}(V)$, hence,

$$
\left[\operatorname{Soc}_{\Lambda} M(I, \tilde{v})=\operatorname{Soc}_{\Lambda} \mathcal{A}(V) / \mathcal{A} v\right] \in \hat{\Lambda}(\text { holonomic }) \backslash \hat{C} \otimes \hat{A} ;
$$

2. let $J$ be a left ideal of $\Lambda$ which contains $\Lambda \cap \mathcal{A}(I, \tilde{v})$ and $J / \Lambda \cap \mathcal{A}(I, \tilde{v})=$ $\operatorname{Soc}_{\Lambda} M(I, \tilde{v})$. Then the left ideal $\mathbf{a}:=J \cap C$ of $C$ is nonzero and for any nonzero element $a \in \mathbf{a}$,

$$
\left[\operatorname{Soc}_{\Lambda} M(I, \tilde{v}) \simeq \Lambda / \Lambda \cap \mathcal{A}(I, \tilde{v}) a^{-1}\right] \in \hat{\Lambda}(\text { holonomic }) \backslash \hat{C} \otimes \hat{A} .
$$

So, any element of $\hat{\Lambda}$ (holonomic) $\backslash \hat{C} \otimes \hat{A}$ is an isoclass of some $\Lambda$-module $\Lambda / \Lambda \cap \mathcal{A}(I, \tilde{v}) a^{-1}$ (for some $I, \tilde{v}$ and a as above). Two such simple $\Lambda$-modules are isomorphic, $\Lambda / \Lambda \cap \mathcal{A}(I, \tilde{v}) a^{-1} \simeq \Lambda / \Lambda \cap \mathcal{A}\left(I_{*}, \tilde{v_{*}}\right) a_{*}^{-1}$, iff the simple $\mathcal{A}$ modules $\mathcal{A} / \mathcal{A}(I, \tilde{v})$ and $\mathcal{A} / \mathcal{A}\left(I_{*}, \tilde{v_{*}}\right)$ are isomorphic.

Proof. 1. Evident.

2. It follows from Theorem 4.8 and Lemma 2.7.

\section{The simple holonomic $\mathcal{D}\left(D_{1} \otimes D_{2}\right)$-modules}

Let $K$ be an algebraically closed field of characteristic zero and let the algebra

$$
\Lambda=C \otimes A
$$

be the tensor product of rings of differential operators with coefficients from a regular commutative affine domain of Krull dimension 1: $C=\Delta\left(D_{1}\right)$ and $A=$ $\Delta\left(D_{2}\right), D=D_{2}$. The algebra $\Lambda$ is isomorphic to the ring of differential operators $\Delta\left(D_{1} \otimes D_{2}\right)$ (Lemma 2.5). We keep the notation from Section 4. In this section the simple holonomic $\Lambda$-modules will be described.

Repeating the same argument (as in Section 4) for the ring $\Lambda$ we have the partition (4.6) and Proposition 4.3 is true, hence there is the partition

$$
\hat{\Lambda}(\text { holonomic })=\hat{C} \otimes \hat{A} \cup \hat{\Lambda} \text { (holonomic) } \backslash \hat{C} \otimes \hat{A} \text {. }
$$

The sets $\hat{C}$ and $\hat{A}$ were described in Section 3. So, in order to finish the classification of $\hat{\Lambda}$ (holonomic) it remains to describe $\hat{\Lambda}$ (holonomic) $\backslash \hat{C} \otimes \hat{A}$. We keep notation of Section 3 for the algebra $A \equiv \Delta(D)$ (substituting the letter $A$ for $\Delta$ ). Fix an element $c \in D$ as in Lemma 2.1.(1) applied to the ring $A=\Delta(D)$, i.e.

$$
A_{c}=D_{c}[X ; \delta]
$$

is an Ore extension for some $X$ and some derivation $\delta$. The algebras $A$ and $A_{c}$ are subalgebras of the algebra $B=l[X ; \delta]$ which is the localization of $A$ at $D_{*}=D \backslash\{0\}$ or the localization of $A_{c}$ at $D_{c} \backslash\{0\}$. We have the inclusion

$$
\Lambda=C \otimes A \subseteq \Lambda_{c}=C \otimes A_{c}
$$


of algebras. Observe that the algebra $\Lambda_{c}$ is of the type considered in Section 4, so the simple holonomic $\Lambda_{c}$-modules are described. Recall that $k$ is the full quotient ring of $C$. The algebra

$$
\mathcal{A}:=C_{*}^{-1} \Lambda_{c}=k \otimes A_{c}=k \otimes D_{c}[X ; \delta]=\mathcal{D}_{c}[X ; \delta],
$$

is the Ore extension where $\mathcal{D}=k \otimes D, \mathcal{D}_{c}=k \otimes D_{c}$.

Theorem 5.1. The map

$$
\hat{\Lambda}(\text { holonomic }) \backslash \hat{C} \otimes \hat{A} \rightarrow \hat{\Lambda}_{c}(\text { holonomic }) \backslash \hat{C} \otimes \hat{A}_{c},[M] \rightarrow\left[M_{c}\right],
$$

is bijective with inverse $[N] \rightarrow\left[\operatorname{Soc}_{\Lambda} N\right]$.

Proof. Observe that if $[M] \in \hat{\Lambda}$ (holonomic) $\backslash \hat{C} \otimes \hat{A}$, then $M_{c} \neq 0$ (otherwise, $[M] \in$ $\hat{C} \otimes \hat{A}$, since $c \in A$ ) and, by Proposition 2.3, $M_{c}$ is a simple holonomic $\Lambda_{c}$-module. Since $\operatorname{Soc}_{C} M=0$ (otherwise, $[M] \in \hat{C} \otimes \hat{A}$ ) and $\operatorname{Soc}_{C} L \neq 0$ for every $[L] \in \hat{C} \otimes \hat{A}_{c}$, we conclude that $\left[M_{c}\right] \in \hat{\Lambda}_{c}$ (holonomic) $\backslash \hat{C} \otimes \hat{A}_{c}$, i.e. the map (5.1) is well-defined.

To finish the proof it suffices to show that each $[N] \in \hat{\Lambda}_{c}$ (holonomic) $\backslash \hat{C} \otimes \hat{A}_{c}$ has nonzero $\operatorname{Soc}_{\Lambda} N$. If we take any nonzero cyclic $\Lambda$-submodule, say $L$, of $N$, then $N=L_{c}$. By Proposition 2.3, $L$ is a holonomic $\Lambda$-module, hence, $L$ contains a simple $\Lambda$-submodule. i.e. $\operatorname{Soc}_{\Lambda} N \neq 0$.

Corollary 5.2. Let $M$ be a nonzero simple $\Lambda$-module and let $\tilde{M}=\mathcal{A} \otimes_{\Lambda} M$. Then

1. $\tilde{M}=0 \Leftrightarrow[M] \in \hat{C} \otimes \hat{A}$;

2. $1 \leq \operatorname{dim}_{k} \tilde{M}<\infty \Leftrightarrow[M] \in \hat{\Lambda}($ holonomic $) \backslash \hat{C} \otimes \hat{A}$;

3. $\operatorname{dim}_{k} \tilde{M}=\infty \Leftrightarrow[M] \in \hat{\Lambda}$ (non-holonomic $)$.

Hence, $M$ is holonomic (resp. non-holonomic) iff $\operatorname{dim}_{k} \tilde{M}<\infty$ (resp. $M_{c}$ contains a free $C \otimes K[X]$-module of rank 1$)$.

Proof. It follows from Theorem 5.1 and Corollary 4.11.

There are natural algebra monomorphisms:

$$
\Lambda=C \otimes A \rightarrow \Lambda_{c}=C \otimes A_{c} \rightarrow \mathcal{A}=k \otimes A_{c}=\mathcal{D}_{c}[X ; \delta] .
$$

Observe that the algebra $\mathcal{A}$ is the localization $\mathcal{A}=S^{-1} \Lambda$ of $\Lambda$ at the Ore subset $S=\bigcup_{j=0}^{\infty} C_{*} c^{j}$ of $\Lambda$. The next corollary follows immediately from Theorem 5.1, Corollary 4.12 and Lemma 2.7.

Corollary 5.3. Let $\left[V=\mathcal{D}_{c} / I\right] \in \hat{\mathcal{D}}_{c}$ for a maximal left ideal $I$ of $\mathcal{D}_{c}$ such that the $\mathcal{A}$-module $\mathcal{A}(V) \equiv \mathcal{A} / \mathcal{A} I$ is not simple and let $v=\tilde{v}+\mathcal{A} I(\tilde{v} \in \mathcal{A})$ be an irreducible element of the ring $\mathcal{E}=\mathcal{E}(\mathcal{A} / \mathcal{A} I)$. Then

1. the $\Lambda$-module

$$
M(I, \tilde{v}):=\Lambda / \Lambda \cap \mathcal{A}(I, \tilde{v})
$$

is a $\Lambda$-submodule of $\mathcal{A}(V)$, hence,

$$
\left[\operatorname{Soc}_{\Lambda} M(I, \tilde{v})=\operatorname{Soc}_{\Lambda} \mathcal{A}(V) / \mathcal{A} v\right] \in \hat{\Lambda}(\text { holonomic }) \backslash \hat{C} \otimes \hat{A}
$$

2. let $J$ be a left ideal of $\Lambda$ which contains $\Lambda \cap \mathcal{A}(I, \tilde{v})$ and $J / \Lambda \cap \mathcal{A}(I, \tilde{v})=$ $\operatorname{Soc}_{\Lambda} M(I, \tilde{v})$. Then the set $\mathbf{a}:=J \cap S$ of $S=\bigcup_{j=0}^{\infty} C_{*} c^{j}$ is non-empty and for any element $a \in \mathbf{a}$,

$$
\left[\operatorname{Soc}_{\Lambda} M(I, \tilde{v}) \simeq \Lambda / \Lambda \cap \mathcal{A}(I, \tilde{v}) a^{-1}\right] \in \hat{\Lambda}(\text { holonomic }) \backslash \hat{C} \otimes \hat{A}
$$


So, any element of $\hat{\Lambda}$ (holonomic $) \backslash \hat{C} \otimes \hat{A}$ is an isoclass of some $\Lambda$-module $\Lambda / \Lambda \cap \mathcal{A}(I, \tilde{v}) a^{-1}$ for some $I, \tilde{v}$ and a as above. Two such simple $\Lambda$-modules are isomorphic, $\Lambda / \Lambda \cap \mathcal{A}(I, \tilde{v}) a^{-1} \simeq \Lambda / \Lambda \cap \mathcal{A}\left(I_{*}, \tilde{v}_{*}\right) a_{*}^{-1}$, iff the simple $\mathcal{A}$ modules $\mathcal{A} / \mathcal{A}(I, \tilde{v})$ and $\mathcal{A} / \mathcal{A}\left(I_{*}, \tilde{v}_{*}\right)$ are isomorphic.

\section{A holonomicity CRiterion of $\mathcal{D}(R)$-modules ( $R$ is Regular of KRull DimENSION 2)}

Let $R$ be a regular commutative affine domain over the algebraically closed field $K$ of characteristic zero. Let $\Delta(R)=\mathcal{D}(R)$ be the ring of differential operators with coefficients from $R$. For the first Weyl algebra $A_{1}=A_{1}(K)$ we denote by $k$ its full quotient ring (the first Weyl skew field). In this section we give a criterion (Theorem 6.1) when a finitely generated $\Delta(R)$-module is holonomic in terms of finite dimensionality of some left vector spaces over the first Weyl skew field.

Fix

$$
\Delta(R) \rightarrow \prod_{i=1}^{s} \Delta(R)_{c_{i}}, \quad c_{i} \in R, i=1, \ldots, s
$$

a faithfully flat extension as in Lemma 2.1. Every ring

$$
\Delta(R)_{c_{i}}=\Delta\left(R_{c_{i}}\right)=R_{c_{i}}\left[X_{i, 1}, \partial / \partial Y_{i, 1}\right]\left[X_{i, 2}, \partial / \partial Y_{i, 2}\right]
$$

is an iterated Ore extension (as in Lemma 2.1) and contains the second Weyl algebra $A_{2}^{(i)}$ :

$$
\begin{gathered}
\Delta(R)_{c_{i}} \supseteq A_{2}^{(i)}:=C^{(i)} \otimes A^{(i)}, C^{(i)}=K\left[Y_{i, 1}\right]\left[X_{i, 1}, \partial / \partial Y_{i, 1}\right], \\
A^{(i)}=K\left[Y_{i}\right]\left[X_{i}, \partial / \partial Y_{i}\right], X_{i}=X_{i, 2}, Y_{i}=Y_{i, 2} .
\end{gathered}
$$

The algebras $\left\{A^{(i)}, C^{(i)}\right\}$ are isomorphic to the first Weyl algebra. Denote by $k_{i}=\left(C_{*}^{(i)}\right)^{-1} C^{(i)}$ the Weyl skew field associated with $C^{(i)}$. Note the second Weyl algebra $A_{2}^{(i)}=C^{(i)} \otimes A^{(i)}$ is the example of the algebra $\Lambda=C \otimes A$ from Section 4 .

The localization $\mathcal{A}^{(i)}$ of $A_{2}^{(i)}$ at $C_{*}^{(i)}$ is the Ore extension

$$
\mathcal{A}^{(i)}=k_{i} \otimes A^{(i)}=k_{i} \otimes K\left[Y_{i}\right]\left[X_{i}, \delta_{i}=\partial / \partial Y_{i}\right] .
$$

Theorem 6.1. Let $M$ be a finitely generated $\Delta(R)$-module. Then

1. the $\Delta(R)$-module $M$ is holonomic $\Leftrightarrow \operatorname{dim}_{k_{i}} \mathcal{A}^{(i)} \otimes_{A_{2}^{(i)}} M_{c_{i}}<\infty$ for $i=$ $1, \ldots, s$;

2. the $\Delta(R)$-module $M$ is non-holonomic $\Leftrightarrow i$ exists such that $\operatorname{dim}_{k_{i}} \mathcal{A}^{(i)} \otimes_{A_{2}^{(i)}}$ $M_{c_{i}}=\infty \Leftrightarrow$ there exists a free $C^{(i)} \otimes K\left[Y_{i}\right]$-submodule of $M_{c_{i}}$ of rank 1 for some $i$.

Proof. It follows immediately from Theorem 2.4(2), (3) and Corollary 4.11.

\section{REFERENCES}

[Bav1] V. V. Bavula, Generalized Weyl algebras and their representations, Algebra i Analiz, 4 (1992), no. 1, 75-97; English transl. in St. Petersburg Math. J. 4 (1993), no. 1, 71-92. MR 93h:16043

[Bav2] V. V. Bavula, Generalized Weyl algebras, kernel and tensor-simple algebras, their simple modules, Representations of algebras. Sixth International Conference, August 1922, 1992. CMS Conference proceedings (V.Dlab and H.Lenzing, Eds.), v. 14, 83-106. MR 94i:16016 
[Bav3] V. V. Bavula, Simple modules of the Ore extensions with Dedekind coefficients, Comm. in Algebra, 27 (1999), no. 6, 2665-2699. MR 2000i:16050

[Bav4] V. V. Bavula, Identification of the Hilbert function and Poincaré series, and the dimension of modules over filtered rings, Russian Acad. Sci. Izv. Math., 44 (1995), no. 2, 225-246. MR 95j:13013

[Bav5] V. V. Bavula, Each Schurian algebra is tensor simple, Comm. in Algebra 23 (1995), no. 4, 1363-1367. MR 96i:16008

[BeLu] I. Bernstein and V. Lunts, On non-holonomic irreducible $\mathcal{D}$-modules, Inv. Math., 94 (1988), 223-243. MR 90b:58247

[Bj] J.-E. Björk, "Rings of differential operators", North Holland, Amsterdam, 1979. MR 82g:32013

[Bl1] R. E. Block, Classification of the irreducible representations of $\operatorname{sl}(2, \mathbf{C})$, Bull. Amer. Math. Soc., 1 (1979), 247-250. MR 80i:17008

[B12] R. E. Block, The irreducible representations of the Weyl algebra $A_{1}$, in "Séminaire d'Algébre Paul Dubreil (Proceedings, Paris 1977-1978)" (M. P. Malliavin, Ed.), Lecture Notes in Mathematics no. 740, pp. 69-79, Springer-Verlag, Berlin, New York, 1979. MR 81e: 17010

[B13] R. E. Block, The irreducible representations of the Lie algebra $\operatorname{sl}(2)$ and of the Weyl algebra, Adv. in Math. 39 (1981), 69-110. MR 83c:17010

[Bor] A. Borel et al., "Algebraic D-modules", Perspectives in Mathematics (J. Coates and S. Helgason, Eds.), vol. 2, Acad. Press, 1987. MR 89g:32014

[BVO1] V. V. Bavula and F. Van Oystaeyen, The simple modules of certain generalized crossed products, J. of Algebra 194 (1997), 521-566. MR 98e:16006

[BVO2] V. V. Bavula and F. Van Oystaeyen, Simple holonomic modules over the second Weyl algebra $A_{2}$, Adv. in Math., 150 (2000), 80-116. CMP 2000:09

[Co] S. C. Coutinho, $d$-simple rings and simple $\mathcal{D}$-modules, Math. Proc. Camb. Phil. Soc. 125 (1999). 405-415. MR 99j:16013

[Jac] N. Jacobson, "The Theory of Rings", Amer. Math. Soc., Providence, R.I., 1943. MR 5:31f

[KL] G. R. Krause and T. H. Lenagan, "Growth of algebras and Gelfand-Kirillov dimension", Revised edition, Graduate Studies in Math., 22, Amer. Math. Soc., Providence, R.I. MR 2000j:16035

[LVO] Li Huishi and F. Van Oystaeyen, "Zariskian Filtratons", Kluwer, 1996. MR 97m:16083

[Lu] V. Lunts, Algebraic varieties preserved by generic flows, Duke Math. J., 58 (1989), no. 3, 531-554. MR 91a:32015

[MR] J. C. McConnell and J. C. Robson, "Noncommutative Noetherian rings", Wiley, 1987. MR 89j: 16023

[St] J. T. Stafford, Non-holonomic modules over Weyl algebras and enveloping algebras, Invent. Math., 79 (1985), 619-638. MR 86h:17009

Department of Pure Mathematics, University of Sheffield, Hicks Building, Sheffield S3 7RH, UK

E-mail address: vbavula@sheffield.ac.uk

E-mail address: bavula@uia.ua.ac.be

Department of Mathematics and Computer Science, University of Antwerp (U.I.A), Universiteitsplein, 1, B-2610, Wilrijk, Belgium

E-mail address: francin@uia.ua.ac.be 